\title{
Iterative properties of birational rowmotion I: generalities and skeletal posets
}

\author{
Darij Grinberg* \\ Department of Mathematics \\ Massachusetts Institute of Technology \\ Massachusetts, U.S.A. \\ darijgrinberg@gmail.com
}

\author{
Tom Roby ${ }^{\dagger}$ \\ Department of Mathematics \\ University of Connecticut \\ Connecticut, U.S.A. \\ tom.roby@uconn.edu
}

Submitted: May 1, 2014; Accepted: Feb 7, 2016; Published: Feb 19, 2016

Mathematics Subject Classifications: 06A07, 05E99

\begin{abstract}
We study a birational map associated to any finite poset $P$. This map is a farreaching generalization (found by Einstein and Propp) of classical rowmotion, which is a certain permutation of the set of order ideals of $P$. Classical rowmotion has been studied by various authors (Fon-der-Flaass, Cameron, Brouwer, Schrijver, Striker, Williams and many more) under different guises (Striker-Williams promotion and Panyushev complementation are two examples of maps equivalent to it). In contrast, birational rowmotion is new and has yet to reveal several of its mysteries. In this paper, we set up the tools for analyzing the properties of iterates of this map, and prove that it has finite order for a certain class of posets which we call "skeletal". Roughly speaking, these are graded posets constructed from one-element posets by repeated disjoint union and "grafting onto an antichain"; in particular, any forest having its leaves all on the same rank is such a poset. We also make a parallel analysis of classical rowmotion on this kind of posets, and prove that the order in this case equals the order of birational rowmotion.
\end{abstract}

Keywords: rowmotion; posets; order ideals; trees; graded posets; tropicalization.

\footnotetext{
*Supported by NSF grant 1001905.

†Supported by NSF grant 1001905.
} 


\section{Contents}

1 Introduction $\quad 2$

1.1 Leitfaden . . . . . . . . . . . . . . . . . . . . . 5 5

2 Linear extensions of posets 5

3 Birational rowmotion $\quad 6$

4 Graded posets $\quad 14$

5 w-tuples $\quad 16$

$\begin{array}{lll}6 & \text { Graded rescaling of labellings } & 17\end{array}$

7 Homogeneous labellings $\quad 19$

8 Order 24

9 The opposite poset $\quad 25$

10 Skeletal posets $\quad 26$

11 Postscript: Classical rowmotion on skeletal posets 32

\section{Introduction}

The present paper, and its continuation [GrRo14], had originally been intended as companion papers to David Einstein's and James Propp's work [EiPr13, EiPr14], which introduced piecewise-linear and birational rowmotion as extensions of the classical concept of rowmotion on order ideals. While the present paper is mathematically self-contained, it provides only a modicum of motivation and applications for the results it discusses. For the latter, the reader may consult [EiPr13].

Let $P$ be a finite poset, and $J(P)$ the set of the order ideals ${ }^{1}$ of $P$. Rowmotion is a classical map $J(P) \rightarrow J(P)$ which can be defined in various ways, one of which is as follows: For every $v \in P$, let $\mathbf{t}_{v}: J(P) \rightarrow J(P)$ be the map sending every order ideal $S \in J(P)$ to

$$
\left\{\begin{array}{l}
S \cup\{v\}, \text { if } v \notin S \text { and } S \cup\{v\} \in J(P) ; \\
S \backslash\{v\}, \text { if } v \in S \text { and } S \backslash\{v\} \in J(P) ; \\
S, \text { otherwise. }
\end{array}\right.
$$

These maps $\mathbf{t}_{v}$ are called classical toggles ${ }^{2}$, since all they do is "toggle" an element into or out of an order ideal. Let $\left(v_{1}, v_{2}, \ldots, v_{m}\right)$ be a linear extension of $P$ (see Definition

\footnotetext{
${ }^{1}$ An order ideal of a poset $P$ is a subset $S$ of $P$ such that every $s \in S$ and $p \in P$ with $p \leqslant s$ satisfy $p \in S$.

${ }^{2}$ or just toggles in literature which doesn't occupy itself with birational rowmotion
} 
2 for the meaning of this). Then, (classical) rowmotion is defined as the composition $\mathbf{t}_{v_{1}} \circ \mathbf{t}_{v_{2}} \circ \ldots \circ \mathbf{t}_{v_{m}}$ (which turns out to be independent of the choice of linear extension $\left.\left(v_{1}, v_{2}, \ldots, v_{m}\right)\right)$. This rowmotion map has been studied from various perspectives; in particular, it is isomorphic ${ }^{3}$ to the map $f$ of Fon-der-Flaass [Flaa93] ${ }^{4}$, the map $F^{-1}$ of Brouwer and Schrijver [BrSchr74], and the map $f^{-1}$ of Cameron and Fon-der-Flaass $[\mathrm{CaFl} 5]^{5}$. More recently, it has been studied (and christened "rowmotion") in Striker and Williams [StWi11], where further sources and context are also given. Since so much has already been said about this rowmotion map, we will only briefly touch on its properties in Section 11, while most of this paper will be spent studying a much more general construction.

Among the questions that have been posed about rowmotion, the most prevalent was probably that of its order: While it clearly has finite order (being a bijective map from the finite set $J(P)$ to itself), it turns out to have a much smaller order than one would naively expect when the poset $P$ has certain "special" forms (e.g., a rectangle, a root poset, a product of a rectangle with a 2-chain, or - apparently first considered in this paper - a forest). Most strikingly, when $P$ is the rectangle $[p] \times[q]$, then the $(p+q)$-th power of the rowmotion operator is the identity map. This is proven in [BrSchr74, Theorem 3.6] and [Flaa93, Theorem 2], and a proof can also be constructed from the ideas given in [PrRo13, §3.3.1]. In Section 11 we give a simple algorithm to find the order of rowmotion on graded forests and similar posets.

In [EiPr13], David Einstein and James Propp (inspired by work of Arkady Berenstein and Anatol Kirillov) have lifted the rowmotion map from the set $J(P)$ of order ideals to the progressively more general setups of:

(a) the order polytope $\mathcal{O}(P)$ of the poset $P$ (as defined in [Stan11, Example 4.6.17] or [Stan86, Definition 1.1]), and

(b) even more generally, the affine variety of $\mathbb{K}$-labellings of $P$ for $\mathbb{K}$ an arbitrary infinite field.

In case (a), order ideals of $P$ are replaced by points in the order polytope $\mathcal{O}(P)$, and the role of the map $\mathbf{t}_{v}$ (for a given $v \in P$ ) is assumed by the map which reflects the $v$-coordinate of a point in $\mathcal{O}(P)$ around the midpoint of the interval of all values it could take without the point leaving $\mathcal{O}(P)$ (while all other coordinates are considered fixed). The operation of "piecewise linear" rowmotion is still defined as the composition of these reflection maps in the same way as rowmotion is the composition of the toggles $\mathbf{t}_{v}$. This "piecewise linear" rowmotion extends (interpolates, even) classical rowmotion, as order ideals correspond to the vertices of the order polytope $\mathcal{O}(P)$ (see [Stan86, Corollary 1.3]). We will not study case (a) here, since all of the results we could find in this case can be

\footnotetext{
${ }^{3}$ By this, we mean that there exists a bijection $\phi$ from $J(P)$ to the set of all antichains of $P$ such that rowmotion is $\phi^{-1} \circ f \circ \phi$.

${ }^{4}$ Indeed, let $\mathcal{A}(P)$ denote the set of all antichains of $P$. Then, the map $J(P) \rightarrow \mathcal{A}(P)$ which sends every order ideal $I \in J(P)$ to the antichain of the maximal elements of $I$ is a bijection which intertwines rowmotion and Fon-der-Flaass' map $f$.

${ }^{5}$ This time, the intertwining bijection from rowmotion to the map $f^{-1}$ of [CaFl95] is given by mapping every order ideal $I$ to its indicator function. This is a bijection from $J(P)$ to the set of Boolean monotonic functions $P \rightarrow\{0,1\}$.
} 
obtained by tropicalization from similar results for case (b).

In case (b), instead of order ideals of $P$ one considers maps from the poset $\widehat{P}:=$ $\{0\} \oplus P \oplus\{1\}$ (where $\oplus$ stands for the ordinal sum; see Definition 7 ) to a given infinite field $\mathbb{K}$ (or, to speak more graphically, of all labellings of the elements of $P$ by elements of $\mathbb{K}$, along with two additional labels "at the very bottom" and "at the very top"). The maps $\mathbf{t}_{v}$ are then replaced by certain birational maps which we call birational v-toggles (Definition 11); the resulting composition is called birational rowmotion and denoted by $R$. By a careful limiting procedure (the tropical limit), we can "degenerate" $R$ to the "piecewise linear" rowmotion of case (a), and thus it can be seen as an even higher generalization of classical rowmotion. We refer to the body of this paper for precise definitions of these maps. Note that birational $v$-toggles (but not birational rowmotion) in the case of a rectangle poset have also appeared in [OSZ13, (3.5)], but (apparently) have not been composed there in a way that yields birational rowmotion.

As in the case of classical rowmotion on $J(P)$, the most interesting question is the order of this map $R$, which in general no longer has an obvious reason to be finite (since the affine variety of $\mathbb{K}$-labellings is not a finite set like $J(P)$ ). Indeed, for some posets $P$ this order is infinite (examples of these can be found in [GrRo14, §12]). In this paper we will prove the following facts:

- Birational rowmotion (i.e., the map $R$ ) on any graded poset (in the meaning of this word introduced in Definition 26) has a very simple effect (namely, cyclic shifting) on the so-called "w-tuple" of a labelling (a rather simple fingerprint of the labelling). This does not mean $R$ itself has finite order (but turns out to be crucial in proving this in several cases).

- Birational rowmotion on graded forests and, slightly more generally, skeletal posets (Definition 69) has finite order (which can be bounded from above by an iterative lcm, and also easily computed algorithmically). Moreover, its order in these cases coincides with the order of classical rowmotion (Section 11).

The following results will be proven in the second paper [GrRo14]:

- Birational rowmotion on a $p \times q$-rectangle has order $p+q$ and satisfies a further symmetry property. These results were originally conjectured by James Propp and the second author, and can be used as an alternative route to certain properties of (Schützenberger's) promotion map on semistandard Young tableaux.

- Birational rowmotion on certain triangle-shaped posets - more precisely, posets which can be obtained from the $p \times p$-square by cutting it along either diagonal, or along both diagonals at once - also has finite order (usually $p$ or $2 p$ ), except for the case when we cut along both diagonals and $p$ is even (we conjecture that the order is $p$ in this case as well).

An extended (12-page) abstract [GrRo13] of this paper and [GrRo14] has been published in the proceedings of the FPSAC 2014 conference. A longer version for readers who would like fuller details and remarks is available on the arXiv [GrRoArX]. 


\section{$1.1 \quad$ Leitfaden}

The Hasse diagram at right shows how the sections of this paper depend upon each other. A section $n$ depends substantially on a section $m$ if and only if $m>n$ in the poset whose Hasse diagram is depicted at right. Only substantial dependencies are shown; dependencies upon definitions do not count as substantial (e.g., Section 9 depends on Definition 59, but this does not make it substantially dependent on Section 8), and dependencies which are only used in proving inessential claims do not count.

No section of this paper depends on the Introduction.

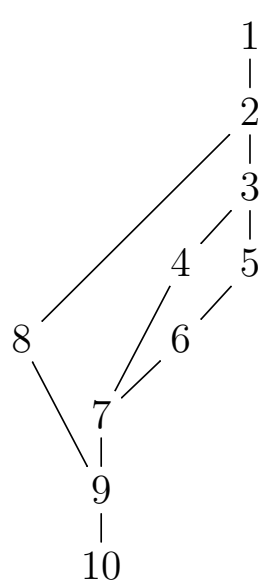

\section{Linear extensions of posets}

This first section serves to introduce some general notions concerning posets and their linear extensions. In particular, we highlight that the set of linear extensions of any finite poset is non-empty and connected by a simple equivalence relation (Proposition 6 ). This will be used in subsequent sections for defining the basic maps that we consider throughout the paper.

We start by defining general notation related to posets:

Definition 1. Let $P$ be a poset. Let $u \in P$ and $v \in P$. In this definition, we will use $\leqslant$, $<, \geqslant$ and $>$ to denote the lesser-or-equal relation, the lesser relation, the greater-or-equal relation and the greater relation, respectively, of the poset $P$.

(a) The elements $u$ and $v$ of $P$ are said to be incomparable if we have neither $u \leqslant v$ nor $u \geqslant v$.

(b) We write $u \lessdot v$ if we have $u<v$ and there is no $w \in P$ such that $u<w<v$. (One often says that " $u$ is covered by $v$ " to signify that $u \lessdot v$.)

(c) We write $u>v$ if we have $u>v$ and there is no $w \in P$ such that $u>w>v$. (Thus, $u \gg v$ holds if and only if $v \lessdot u$.) (One often says that " $u$ covers $v$ " to signify that $u>v$.

(d) An element $u$ of $P$ is called maximal if every $v \in P$ satisfying $v \geqslant u$ satisfies $v=u$. It is easy to see that every nonempty finite poset has at least one maximal element.

(e) An element $u$ of $P$ is called minimal if every $v \in P$ satisfying $v \leqslant u$ satisfies $v=u$. It is easy to see that every nonempty finite poset has at least one minimal element.

When any of these notations becomes ambiguous because the elements involved belong to several different posets simultaneously, we will disambiguate it by adding the words "in $P$ " (where $P$ is the poset which we want to use).

Definition 2. Let $P$ be a finite poset. A linear extension of $P$ will mean a list $\left(v_{1}, v_{2}, \ldots, v_{m}\right)$ of the elements of $P$ such that every element of $P$ occurs exactly once in this list, and such that any $i \in\{1,2, \ldots, m\}$ and $j \in\{1,2, \ldots, m\}$ satisfying $v_{i}<v_{j}$ in $P$ must satisfy $i<j$. 
Theorem 3. Every finite poset $P$ has a linear extension.

Theorem 3 is a well-known fact, and can be proven, e.g., by induction over $|P|$ (with the induction step consisting of splitting off a maximal element $u$ of $P$ and appending it to a linear extension of the residual poset $P \backslash\{u\}$ ).

The following proposition can be easily checked by the reader:

Proposition 4. Let $P$ be a finite poset. Let $\left(v_{1}, v_{2}, \ldots, v_{m}\right)$ be a linear extension of $P$. Let $i \in\{1,2, \ldots, m-1\}$ be such that the elements $v_{i}$ and $v_{i+1}$ of $P$ are incomparable. Then $\left(v_{1}, v_{2}, \ldots, v_{i-1}, v_{i+1}, v_{i}, v_{i+2}, v_{i+3}, \ldots, v_{m}\right)$ (this is the tuple obtained from the tuple $\left(v_{1}, v_{2}, \ldots, v_{m}\right)$ by interchanging the adjacent entries $v_{i}$ and $\left.v_{i+1}\right)$ is a linear extension of $P$ as well.

Definition 5. Let $P$ be a finite poset. The set of all linear extensions of $P$ will be called $\mathcal{L}(P)$. Thus, $\mathcal{L}(P) \neq \varnothing$ (by Theorem 3 ).

In our approach to birational rowmotion, we will use the following fact (which is folklore and has applications in various contexts, including Young tableau theory):

Proposition 6. Let $P$ be a finite poset. Let $\sim$ denote the finest equivalence relation on $\mathcal{L}(P)$ with the following property: For any linear extension $\left(v_{1}, v_{2}, \ldots, v_{m}\right)$ of $P$ and any $i \in\{1,2, \ldots, m-1\}$ such that the elements $v_{i}$ and $v_{i+1}$ of $P$ are incomparable, we have $\left(v_{1}, v_{2}, \ldots, v_{m}\right) \sim\left(v_{1}, v_{2}, \ldots, v_{i-1}, v_{i+1}, v_{i}, v_{i+2}, v_{i+3}, \ldots, v_{m}\right)$ (noting that $\left(v_{1}, v_{2}, \ldots, v_{i-1}, v_{i+1}, v_{i}, v_{i+2}, v_{i+3}, \ldots, v_{m}\right)$ is also a linear extension of $P$, because of Proposition 4).

This proposition is basic (it generalizes the fact that the symmetric group $S_{n}$ is generated by the adjacent-element transpositions) and classical, but a proof is hard to find in the literature. One proof is in [AKSch12, Proposition 4.1 (for the $\pi^{\prime}=\pi \tau_{j}$ case)]; another is sketched in [Rusk92, p. 79] and presented in more detail in [Etienn84, Lemma 1]. We give a fully-detailed proof in [GrRoArX].

\section{Birational rowmotion}

In this section, we introduce the basic objects whose nature we will investigate: labellings of a finite poset $P$ (by elements of a field) and a birational map between them called "birational rowmotion". This map generalizes (in a certain sense) the notion of ordinary rowmotion on the set $J(P)$ of order ideals of $P$ to the vastly more general setting of field-valued labellings. We will discuss the technical concerns raised by the definitions, and provide an example and an alternative description of birational rowmotion. A deeper study of birational rowmotion is deferred to the following sections.

The concepts which we are going to define now go back to [EiPr13] and earlier sources, and are often motivated there. The reader should be warned that the notations used in [EiPr13] are not identical with those used in the present paper (not to mention that [EiPr13] is working over $\mathbb{R}_{+}$rather than over fields as we do). 


\section{Notational conventions used throughout this paper:}

Unless otherwise stated, we will consistently use the following notations.

$\mathbb{N}$ denotes the set of all nonnegative integers.

$P$ will denote a finite poset (although a few definitions apply equally well to infinite posets). In Sections 3-9, $P$ will denote an $n$-graded poset, where $n \in \mathbb{N}$ is a nonnegative integer. (The meaning of this will be defined in Section 3.)

$\mathbb{K}$ will denote a field, which we tacitly assume is either infinite or at least can be enlarged when necessity arises. This assumption is needed in order to clarify the notions of rational maps and generic elements of algebraic varieties over $\mathbb{K}$. We do not require $\mathbb{K}$ to be algebraically closed.

Definition 7 . Let $P$ be a poset. Then, $\widehat{P}$ will denote the poset whose ground set is the disjoint union $P \cup\{0,1\}$, with partial order relation

$$
(a \leqslant b) \Longleftrightarrow(\text { either }(a \in P \text { and } b \in P \text { and } a \leqslant b \text { in } P \text { ) or } a=0 \text { or } b=1)
$$

(where "either/or" has a non-exclusive meaning). Here and in the following, we regard the canonical injection of the set $P$ into the disjoint union $\widehat{P}$ as an inclusion; thus, $P$ becomes a subposet of $\widehat{P}$. In the terminology of Stanley's [Stan11, section 3.2], this poset $\widehat{P}$ is the ordinal sum $\{0\} \oplus P \oplus\{1\}$. Since the relation $<_{P}$ is a restriction of $<_{\widehat{P}}$, we never need to distinguish these from one another.

Definition 8. A $\mathbb{K}$-labelling of $P$ will mean a map $f: \widehat{P} \rightarrow \mathbb{K}$. Thus, $\mathbb{K}^{\widehat{P}}$ is the set of all $\mathbb{K}$-labellings of $P$. If $f$ is a $\mathbb{K}$-labelling of $P$ and $v$ is an element of $\widehat{P}$, then $f(v)$ will be called the label of $f$ at $v$.

Definition 9. We will use the terminology of algebraic varieties and rational maps between them, although the only algebraic varieties that we will be considering are products of affine and projective spaces, as well as their open subsets. We use the punctured arrow $\rightarrow$ to signify rational maps. A rational map $U \rightarrow V$ is said to be dominant if its image is dense in $V$ (with respect to the Zariski topology).

The words generic and almost will always refer to the Zariski topology. For example, if $U$ is a finite set, then an assertion saying that some statement holds "for almost every point $p \in \mathbb{K}^{U}$ " is supposed to mean that there is a Zariski-dense open subset $D$ of $\mathbb{K}^{U}$ such that this statement holds for every point $p \in D$. A "generic" point on an algebraic variety $V$ (for example, this can be a "generic matrix" when $V$ is a space of matrices, or a "generic $\mathbb{K}$-labelling of a poset $P$ " when $V$ is the space of all $\mathbb{K}$-labellings of $P$ ) means a point lying in some fixed Zariski-dense open subset $S$ of $V$; the concrete definition of $S$ can usually be inferred from the context (often, it will be the subset of $V$ on which everything we want to do with our point is well-defined), but of course should never depend on the actual point. We will sometimes abuse notation and say that an equality holds "for every point" instead of "for almost every point" when it is really clear what the $S$ is. (For example, if we say that "the equality $\frac{x^{3}-y^{3}}{x-y}=x^{2}+x y+y^{2}$ holds for every $x \in \mathbb{K}$ and $y \in \mathbb{K}$ ", it is clear that $S$ has to be the set $\mathbb{K}^{2} \backslash\left\{(x, y) \in \mathbb{K}^{2} \mid x=y\right\}$, because the left hand side of the equality makes no sense when $(x, y)$ is outside of this set.) 
Remark 10. Most statements that we make below work not only for fields, but also more generally for semifields ${ }^{6}$ such as the semifield $\mathbb{Q}_{+}$of positive rationals or the tropical semiring. Some (but not all!) statements actually simplify when the underlying field is replaced by a semifield in which no two nonzero elements add to zero (because in such cases, e.g., the denominators in (1) cannot become zero unless some labels of $f$ are 0). Thus, working with such semifields instead of fields would save us the trouble of having things defined "almost everywhere". Moreover, applying our results to the tropical semifield would yield some of the statements about order polytopes made in [EiPr13]. Nevertheless, we prefer to work with fields, for the following reasons:

- If we were to work in semifields which do contain two nonzero elements summing up to zero, then we would still have the issue of zero denominators, but we are not aware of a theoretical framework in the spirit of Zariski topology for fields to reassure us in this case that these issues are negligible.

- If an identity between subtraction-free rational expressions (such as $\frac{x^{3}+y^{3}}{x+y}+3 x y=$ $\left.(x+y)^{2}\right)$ holds over every field (as long as the denominators involved are nonzero), then it must hold over every semifield as well (again as long as the denominators involved are nonzero), even if a proof of this identity uses subtraction in its intermediate steps (e.g., a proof of $\frac{x^{3}+y^{3}}{x+y}+3 x y=(x+y)^{2}$ over a field can begin by simplifying $\frac{x^{3}+y^{3}}{x+y}$ to $x^{2}-x y+$ $y^{2}$, a technique not available over a semifield). This is simply because every true identity between subtraction-free rational expressions can be verified by multiplying by a common denominator and comparing coefficients. Since our main results (such as Proposition 71) can be construed as identities between subtraction-free rational expressions, this yields that all these results hold over any semifield (provided the denominators are nonzero) if they hold over every field. So we are not losing any generality by restricting ourselves to considering only fields.

Definition 11. Let $v \in P$. We define a rational map $T_{v}: \mathbb{K}^{\widehat{P}} \rightarrow \mathbb{K}^{\widehat{P}}$ by

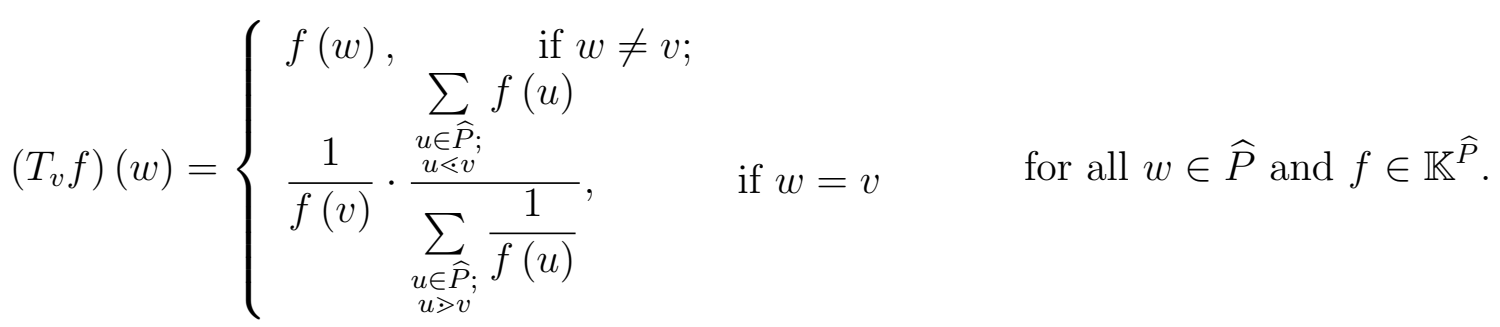

Note that this rational map $T_{v}$ is well-defined, because the right-hand side of (1) is welldefined on a Zariski-dense open subset of $\mathbb{K}^{\widehat{P}}$. (This follows from the fact that for every $v \in P$, there is at least one $u \in \widehat{P}$ such that $u>v$.) We call $T_{v}$ the $v$-toggle.

\footnotetext{
${ }^{6}$ The word "semifield" here means a commutative semiring in which each element other than 0 has a multiplicative inverse. (In contrast to other authors' conventions, our semifields do have zeroes.) A semiring is defined as a set with two binary operations called "addition" and "multiplication" and two elements 0 and 1 which satisfies all axioms of a ring except for having additive inverses.
} 
The map $T_{v}$ that we have just introduced (but defined over the semifield $\mathbb{R}_{+}$instead of our field $\mathbb{K}$ ) is called a "birational toggle operation" in [EiPr13] (where it is denoted by $\phi_{i}$ with $i$ being a number indexing the elements $v$ of $P$; however, the same notation is used for the "tropicalized" version of $T_{v}$ ). As is clear from its definition, it only changes the label at the element $v$. Note also the following almost trivial fact:

Proposition 12. For every $v \in P$, the rational map $T_{v}$ is an involution, i.e., the map $T_{v}^{2}$ is well-defined on a Zariski-dense open subset of $\mathbb{K}^{\widehat{P}}$ and satisfies $T_{v}^{2}=\mathrm{id}$ on this subset.

We are calling this "almost trivial" because one subtlety is easily overlooked: We have to check that the map $T_{v}^{2}$ is well-defined on a Zariski-dense open subset of $\mathbb{K}^{\widehat{P}}$; this requires observing that for every $v \in P$, there exists at least one $u \in \widehat{P}$ such that $u \lessdot v$.

Proposition 12 yields the following:

Corollary 13. For every $v \in P$, the map $T_{v}$ is a dominant rational map.

Dominant rational maps (unlike general rational maps) can be composed, and their compositions are still dominant rational maps. It is very easy to check the following "locality principle":

Proposition 14. For all $v, w \in P$, we have $T_{v} \circ T_{w}=T_{w} \circ T_{v}$, unless either $v \lessdot w$ or $w \lessdot v$.

Proof of Proposition 14. The action of $T_{v}$ on a labelling of $P$ merely changes the label at $v$ to a new value which depends on the label at $v$ and on the labels at the elements $u \in \widehat{P}$ satisfying $u \lessdot v$ or $u>v$. Thus, the actions of $T_{v}$ and $T_{w}$ don't interfere with each other unless either $v \lessdot w$ or $w \lessdot v$ or $v=w$. Hence, $T_{v} \circ T_{w}=T_{w} \circ T_{v}$ unless $v \lessdot w$ or $w \lessdot v$ or $v=w$ (in which latter case, the proposition is obvious).

Corollary 15. Let $v$ and $w$ be two incomparable elements of $P$. Then, $T_{v} \circ T_{w}=T_{w} \circ T_{v}$.

Combining Corollary 15 with Proposition 6, we obtain:

Corollary 16. Let $\left(v_{1}, v_{2}, \ldots, v_{m}\right)$ be a linear extension of $P$. Then, the dominant rational map $T_{v_{1}} \circ T_{v_{2}} \circ \ldots \circ T_{v_{m}}: \mathbb{K}^{\widehat{P}} \rightarrow \mathbb{K}^{\widehat{P}}$ is well-defined and independent of the choice of the linear extension $\left(v_{1}, v_{2}, \ldots, v_{m}\right)$.

Definition 17. Birational rowmotion is defined as the dominant rational map $T_{v_{1}} \circ T_{v_{2}} \circ$ $\ldots \circ T_{v_{m}}: \mathbb{K}^{\widehat{P}} \rightarrow \mathbb{K}^{\widehat{P}}$, where $\left(v_{1}, v_{2}, \ldots, v_{m}\right)$ is a linear extension of $P$. This rational map is well-defined by Corollary 16 and Theorem 3; it will be denoted by $R$.

The reason for the names "birational toggle" and "birational rowmotion" is explained in [EiPr13], in which birational rowmotion (defined over $\mathbb{R}_{+}$rather than over $\mathbb{K}$ ) is denoted (serendipitously from the standpoint of the second author of this paper) by $\rho_{\mathcal{B}}$. 
Example 18. Let us demonstrate the effect of birational toggles and birational rowmotion on a rather simple 4-element poset. Namely, for this example, we let $P$ be the poset $\left\{p, q_{1}, q_{2}, q_{3}\right\}$ with order relation defined by setting $p<q_{i}$ for each $i \in\{1,2,3\}$. This poset has Hasse diagram

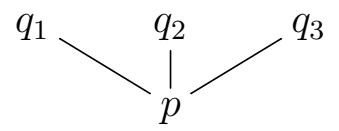

The extended poset $\widehat{P}$ has Hasse diagram

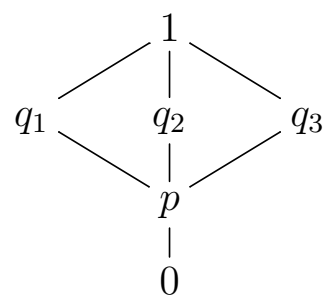

We can visualize a $\mathbb{K}$-labelling $f$ of $P$ by replacing, in the Hasse diagram of $\widehat{P}$, each element $v \in \widehat{P}$ by the label $f(v)$. Let $f$ be a $\mathbb{K}$-labelling sending $0, p, q_{1}, q_{2}, q_{3}$, and 1 to $a, w, x_{1}, x_{2}, x_{3}$, and $b$, respectively (all in $\mathbb{K}$ ); this $f$ is then visualized as follows:

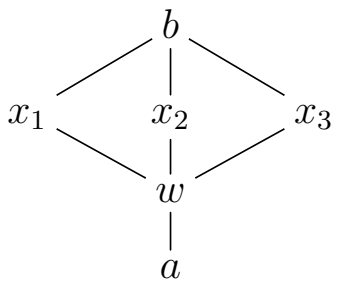

Since $\left(p, q_{1}, q_{2}, q_{3}\right)$ is a linear extension of $P$, we have $R=T_{p} \circ T_{q_{1}} \circ T_{q_{2}} \circ T_{q_{3}}$. Let us track how this transforms our labelling $f$. We first apply $T_{q_{3}}$, obtaining

$$
T_{q_{3}} f=
$$

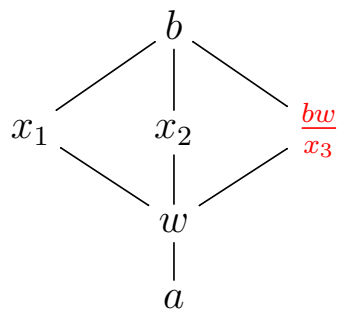

(where we colored the label at $q_{3}$ red to signify that it is the label at the element which got toggled). Indeed, only the label at $q_{3}$ changes under $T_{q_{3}}$ becoming

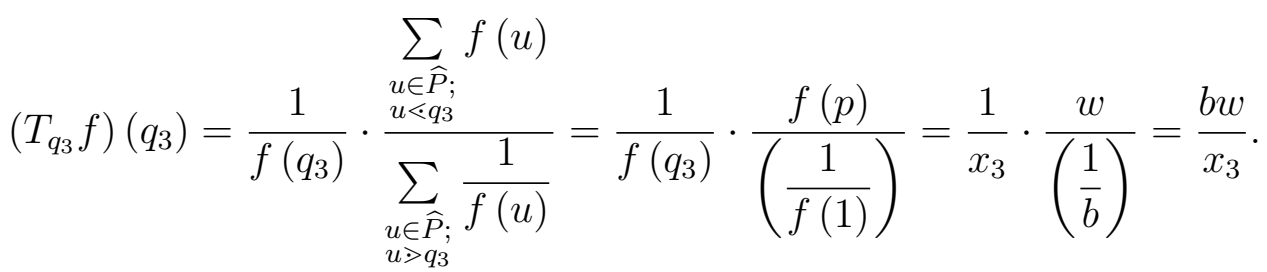


Now applying successively $T_{q_{2}}, T_{q_{1}}$, and $T_{p}$, we obtain

$$
R f=T_{p} T_{q_{1}} T_{q_{2}} T_{q_{3}} f=
$$

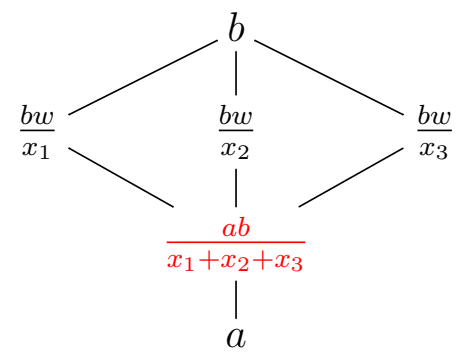

since the birational $p$-toggle $T_{p}$ has changed the label at $p$ to

$$
\begin{aligned}
\left(T_{p} T_{q_{1}} T_{q_{2}} T_{q_{3}} f\right)(p) & =\frac{1}{\left(T_{q_{1}} T_{q_{2}} T_{q_{3}} f\right)(p)} \cdot \frac{\sum_{\substack{u \in \widehat{P} ; \\
u \ll p}}\left(T_{q_{1}} T_{q_{2}} T_{q_{3}} f\right)(u)}{\sum_{\substack{u \in \widehat{P} ; \\
u \gg p}} \frac{1}{\left(T_{q_{1}} T_{q_{2}} T_{q_{3}} f\right)(u)}} \\
& =\frac{1}{w} \cdot \frac{a}{\frac{1}{b w / x_{1}}+\frac{1}{b w / x_{2}}+\frac{1}{b w / x_{3}}}=\frac{a b}{x_{1}+x_{2}+x_{3}} .
\end{aligned}
$$

By repeating this procedure (or just substituting the labels of $R f$ obtained as variables), we can compute $R^{2} f, R^{3} f$ etc. Specifically, we obtain

$$
R^{2} f=
$$

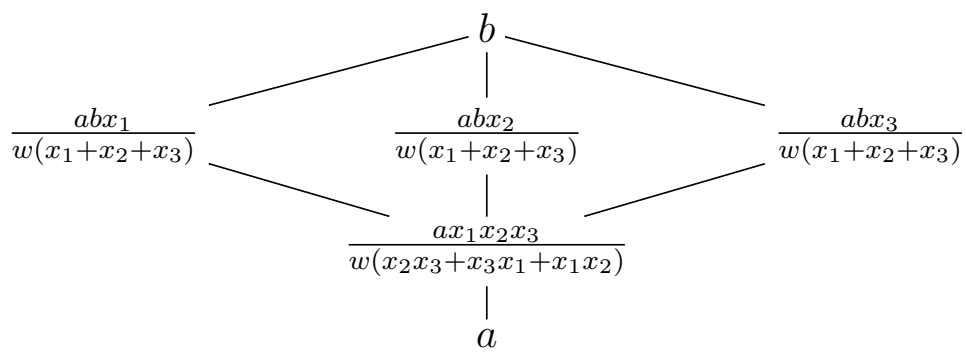

$$
R^{3} f=
$$

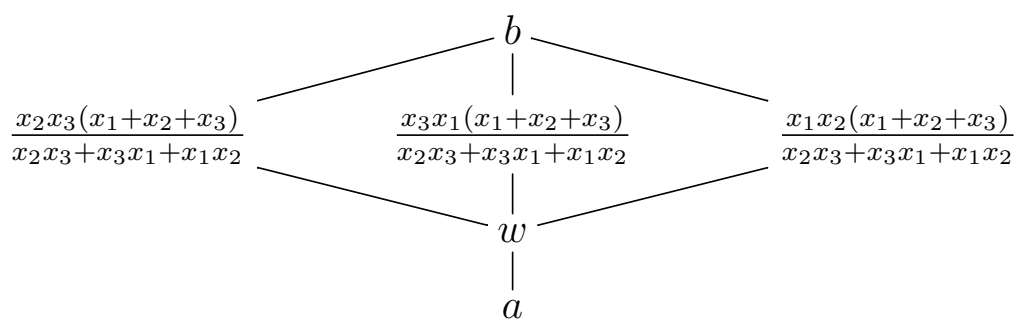



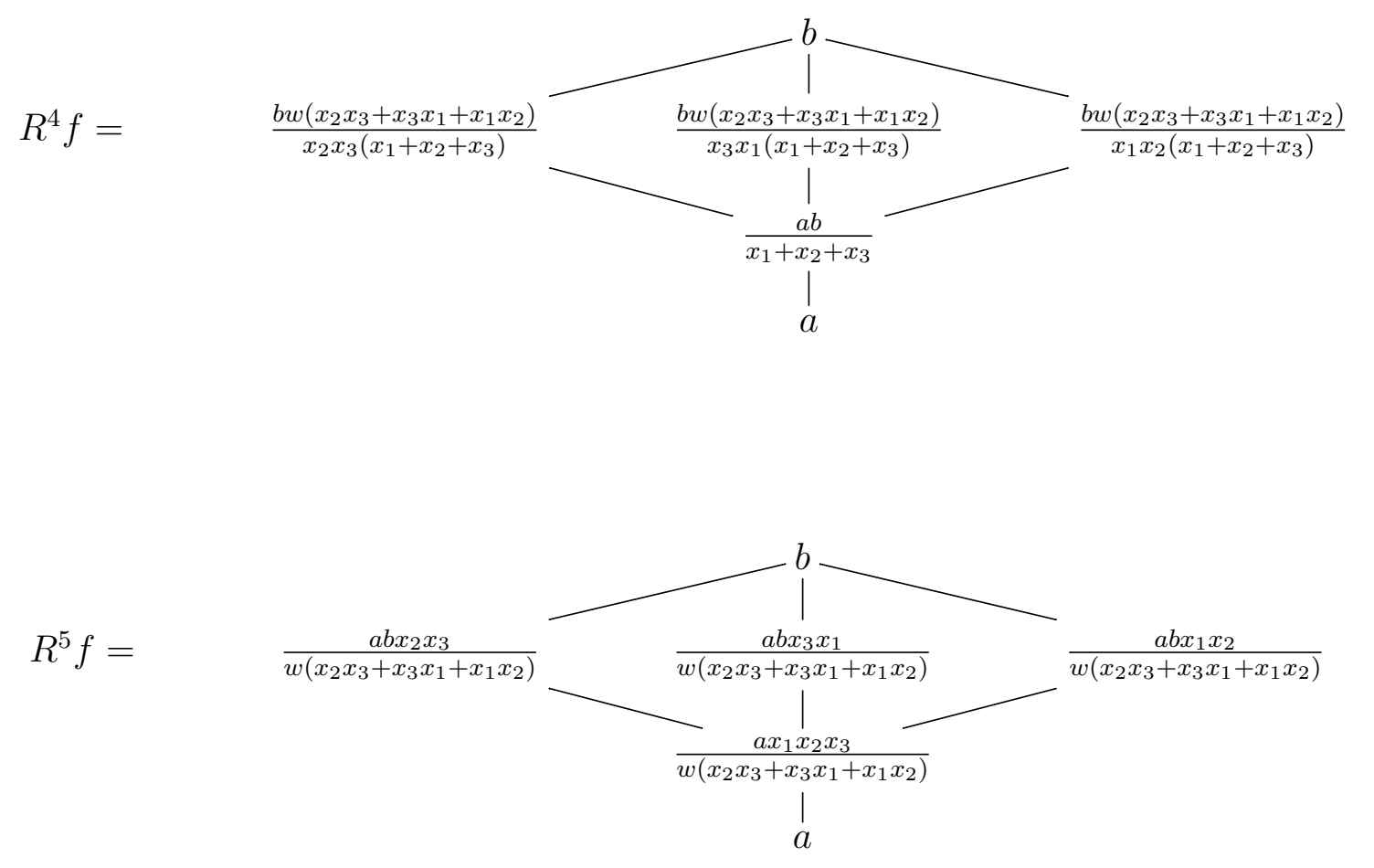

$$
R^{6} f=f
$$

There are several patterns here that catch the eye, some of which are related to the very simple structure of $P$ and don't seem to generalize well. However, the most striking observation here is that $R^{n} f=f$ for some positive integer $n$ (namely, $n=6$ ). We will see in Proposition 71 that this generalizes to a rather wide class of posets, which we call "skeletal posets" (Definition 69), a class of posets which contain (in particular) all graded forests such as our poset $P$ here. A different example is given in [GrRo14].

Let us state another proposition, which describes birational rowmotion implicitly:

Proposition 19. Let $v \in P$ and $f \in \mathbb{K}^{\widehat{P}}$. Then,

$$
(R f)(v)=\frac{1}{f(v)} \cdot \frac{\sum_{\substack{u \in \widehat{P} ; \\ u \ll v}} f(u)}{\sum_{\substack{u \in \widehat{P} ; \\ u \gg v}} \frac{1}{(R f)(u)}} .
$$

Here (and in statements further down this paper), we are taking the liberty to leave assumptions such as "Assume that $R f$ is well-defined" unsaid (for instance, such an assumption is needed in Proposition 19) because these assumptions are satisfied when the parameters belong to some Zariski-dense open subset of their domains. 
Proof of Proposition 19. Fix a linear extension $\left(v_{1}, v_{2}, \ldots, v_{m}\right)$ of $P$. Recall that $R$ has been defined as the composition $T_{v_{1}} \circ T_{v_{2}} \circ \ldots \circ T_{v_{m}}$. Hence, $R f$ can be obtained from $f$ by traversing the linear extension $\left(v_{1}, v_{2}, \ldots, v_{m}\right)$ in the order $v_{m}, v_{m-1}, \ldots$, at every step toggling the element being traversed. When an element $v$ is being toggled, the elements $u \in \widehat{P}$ satisfying $u \lessdot v$ have not yet been toggled, whereas those satisfying $u>v$ have been toggled already. Denoting the state of the $\mathbb{K}$-labelling before the $v$-toggle by $g$, we see that the state after the $v$-toggle will be $T_{v} g$ with

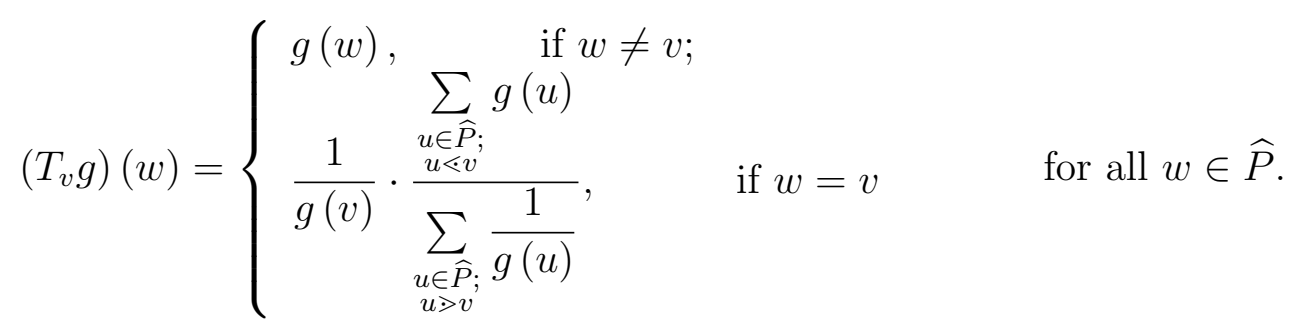

But $g(v)=f(v)$ (since $v$ has not yet been toggled at the time of $g$ ) and $\left(T_{v} g\right)(v)=$ $(R f)(v)$ (since $v$ has been toggled at the time of $T_{v} g$, and is not going to be toggled again during the process of computing $R f$ ); moreover, all $u \in \widehat{P}$ satisfying $u \lessdot v$ satisfy $g(u)=f(u)$ (since these $u$ have not yet been toggled), whereas all $u \in \widehat{P}$ satisfying $u>v$ satisfy $g(u)=(R f)(u)$ (since these $u$ have already been toggled once and for all). Thus, (3) (applied to $w=v$ ) transforms into (2), proving Proposition 19.

Here is a little triviality to complete the picture of Proposition 19:

Proposition 20. Let $f \in \mathbb{K}^{\widehat{P}}$. Then, $(R f)(0)=f(0)$ and $(R f)(1)=f(1)$.

This is clear since no toggle changes the labels at 0 and 1.

We will often use Proposition 20 tacitly. A trivial corollary of Proposition 20 is:

Corollary 21. Let $f \in \mathbb{K}^{\widehat{P}}$. Then for all $\ell \in \mathbb{N}$, we have $\left(R^{\ell} f\right)(0)=f(0)$ and $\left(R^{\ell} f\right)(1)=f(1)$.

We will also need a converse of Propositions 19 and 20:

Proposition 22. Let $f, g \in \mathbb{K}^{\widehat{P}}$ satisfy $f(0)=g(0)$ and $f(1)=g(1)$. Assume that

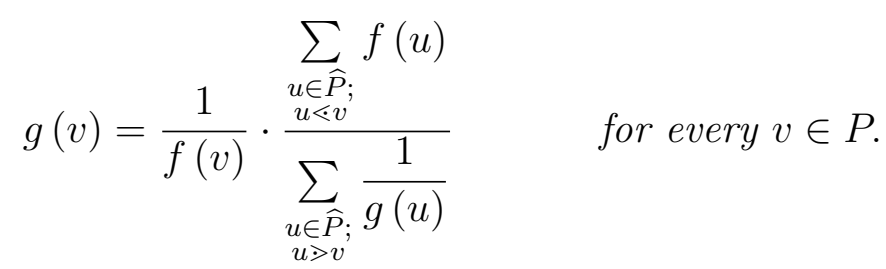

(This means, in particular, that we assume that all denominators in (4) are nonzero.) Then, $R f$ is well-defined and $R f=g$. 
Proof of Proposition 22. It is clearly enough to show that $g(v)=(R f)(v)$ for every $v \in \widehat{P}$. Since this is clear for $v=0$, we only need to consider the case when $v \in\{1\} \cup P$. In this case, we can prove $g(v)=(R f)(v)$ by descending induction over $v$ - that is, we assume as an induction hypothesis that $g(u)=(R f)(u)$ holds for all elements $u \in\{1\} \cup P$ which are greater than $v$ in $\widehat{P}$. The induction base $(v=1)$ is clear, and the induction step follows by comparing (2) with (4). We leave the details (including a check that $R f$ is well-defined, which piggybacks on the induction) to the reader (see also [GrRoArX]).

As an aside, at this point we could give an alternative proof of Corollary 16, foregoing the use of Proposition 6. Indeed, Propositions 19, 20 and 22 characterize $R$ independent of the choice of linear extension. Details are left to the reader.

On a related note, Proposition 19, Proposition 20 and Proposition 22 combined can be used as an alternative definition of birational rowmotion $R$, which works even when the poset $P$ fails to be finite, as long as for every $v \in P$, there exist only finitely many $u \in P$ satisfying $u>v$ and there exist only finitely many $u \in P$ satisfying $u \lessdot v$ (provided that some technicalities arising from Zariski topology on infinite-dimensional spaces are dealt with $)^{7}$. We will not dwell on this.

Another general property of birational rowmotion concerns the question of what happens if the birational toggles are composed not in the "from top to bottom" order as in the definition of birational rowmotion, but the other way round. It turns out that the result is the inverse of birational rowmotion, since we are simply composing involutions in the opposite order.

Proposition 23. Birational rowmotion $R$ on a finite poset $P$ is invertible (as a rational map). Its inverse $R^{-1}$ is $T_{v_{m}} \circ T_{v_{m-1}} \circ \ldots \circ T_{v_{1}}: \mathbb{K}^{\widehat{P}} \rightarrow \mathbb{K}^{\widehat{P}}$, where $\left(v_{1}, v_{2}, \ldots, v_{m}\right)$ is a linear extension of $P$.

\section{Graded posets}

In this section, we restrict our attention to what we call graded posets (a notion that encompasses most of the posets we are interested in; see Definition 24), and define (for this kind of posets) a family of "refined rowmotion" operators $R_{i}$ which toggle only the labels of the $i$-th degree of the poset. These each turn out to be involutions, and their composition from top to bottom degree is $R$ on the entire poset. We will later on use these $R_{i}$ to get a better understanding of $R$ on graded posets.

Let us first introduce our notion of a graded poset:

Definition 24. Let $P$ be a finite poset. Let $n$ be a nonnegative integer. We say that the poset $P$ is $n$-graded if there exists a surjective map deg: $P \rightarrow\{1,2, \ldots, n\}$ such that the following three assertions hold:

Assertion 1: Any two elements $u$ and $v$ of $P$ such that $u>v$ satisfy $\operatorname{deg} u=\operatorname{deg} v+1$.

\footnotetext{
${ }^{7}$ The asymmetry between the $>$ and $\lessdot$ signs in this requirement is intentional. For instance, birational rowmotion can be defined (but will not be invertible) for the poset $\{0,-1,-2,-3, \ldots\}$ (with the usual order relation), but not for the poset $\{0,1,2,3, \ldots\}$ (again with the usual order relation).
} 
Assertion 2: We have $\operatorname{deg} u=1$ for every minimal element $u$ of $P$.

Assertion 3: We have $\operatorname{deg} v=n$ for every maximal element $v$ of $P$.

Example 25. The poset $P$ studied in Example 18 is 2-graded. The empty poset is 0 -graded, but not $n$-graded for any positive $n$. A chain with $k$ elements is $k$-graded.

Definition 26. Let $P$ be a finite poset. We say that the poset $P$ is graded if there exists an $n \in \mathbb{N}$ such that $P$ is $n$-graded.

Definition 27. Let $n \in \mathbb{N}$. Let $P$ be an $n$-graded poset. Then, there exists a surjective map deg $: P \rightarrow\{1,2, \ldots, n\}$ that satisfies the Assertions 1, 2 and 3 of Definition 24. A moment of thought reveals that such a map deg is also uniquely determined by $P \quad 8$. Thus, we will call deg the degree map of $P$.

Moreover, we extend this map deg to a map $\widehat{P} \rightarrow\{0,1, \ldots, n+1\}$ by letting it map 0 to 0 and 1 to $n+1$. This extended map will also be denoted by deg and called the degree map. Notice that this extended map deg still satisfies Assertion 1 of Definition 24 if $P$ is replaced by $\widehat{P}$ in that assertion.

For every $i \in\{0,1, \ldots, n+1\}$, we will denote by $\widehat{P}_{i}$ the subset $\operatorname{deg}^{-1}(\{i\})$ of $\widehat{P}$. For every $v \in \widehat{P}$, the number $\operatorname{deg} v$ is called the degree of $v$.

The notion of an " $n$-graded poset" we just defined is identical with the notion of a "graded finite poset of rank $n-1$ " as defined in [Stan11, §3.1]; in particular, all maximal chains have the same length. (There are several other definitions of "graded" lurking in different corners of combinatorics.) The degree of an element $v$ of $P$ as defined in Definition 27 is off by 1 from the rank of $v$ in $P$ in the sense of [Stan11, §3.1], but the degree $\operatorname{deg} v$ of an element $v$ of $\widehat{P}$ equals its rank in $\widehat{P}$ in the sense of [Stan11, §3.1].

From this point until $\S 10, P$ will always denote an n-graded poset for some (fixed) $n \in \mathbb{N}$.

The way we extended the map deg $: P \rightarrow\{1,2, \ldots, n\}$ to a map deg $: \widehat{P} \rightarrow$ $\{0,1, \ldots, n+1\}$ in Definition 27, of course, was not arbitrary. In fact, it was tailored to make the following (easily proved) statements true:

Proposition 28. Let $u, v \in \widehat{P}$. Consider the map $\operatorname{deg}: \widehat{P} \rightarrow\{0,1, \ldots, n+1\}$ defined in Definition 27.

(a) If $u \lessdot v$ in $\widehat{P}$, then $\operatorname{deg} u=\operatorname{deg} v-1$.

(b) If $u<v$ in $\widehat{P}$, then $\operatorname{deg} u<\operatorname{deg} v$.

(c) If $u<v$ in $\widehat{P}$ and $\operatorname{deg} u=\operatorname{deg} v-1$, then $u \lessdot v$ in $\widehat{P}$.

(d) If $u \neq v$ and $\operatorname{deg} u=\operatorname{deg} v$, then $u$ and $v$ are incomparable in $\widehat{P}$.

Corollary 29. Fix $i \in\{1,2, \ldots, n\}$, and let $\left(u_{1}, u_{2}, \ldots, u_{k}\right)$ be any list of the elements of $\widehat{P}_{i}$ with every element of $\widehat{P}_{i}$ appearing exactly once in the list. Then, the dominant rational map $T_{u_{1}} \circ T_{u_{2}} \circ \ldots \circ T_{u_{k}}: \mathbb{K}^{\widehat{P}} \rightarrow \mathbb{K}^{\widehat{P}}$ is well-defined and independent of the choice of the list $\left(u_{1}, u_{2}, \ldots, u_{k}\right)$.

\footnotetext{
${ }^{8}$ In fact, if $v \in P$, then it is easy to see that $\operatorname{deg} v$ equals the number of elements of any maximal chain in $P$ with highest element $v$. This clearly determines $\operatorname{deg} v$ uniquely.
} 
Proof of Corollary 29. This is analogous to, but simpler than the proof of Corollary 16, because any two distinct elements of $\widehat{P}_{i}$ are incomparable.

Definition 30. Let $n, \mathbb{K}$ and $P$ be as in Corollary 29. Then, we define $R_{i}$ to be the dominant rational map $T_{u_{1}} \circ T_{u_{2}} \circ \ldots \circ T_{u_{k}}: \mathbb{K}^{\widehat{P}} \rightarrow \mathbb{K}^{\widehat{P}}$ described in Corollary 29.

The following two propositions show that for $n$-graded posets $\mathrm{P}$, the $R_{i}$ 's give a way of writing $R$ as a product of fewer (but more complicated than toggles) involutions. The proofs involve simply writing everything as products of toggle maps and noting that each $R_{i}$ is a product of commuting involutions.

Proposition 31. The rowmotion operator on any n-graded poset $P$ can be factored as

$$
R=R_{1} \circ R_{2} \circ \ldots \circ R_{n} .
$$

Proposition 32. For $i \in\{1,2, \ldots, n\}$, the birational map $R_{i}$ is an involution (that is, $R_{i}^{2}=\mathrm{id}$ on the set where $R_{i}$ is defined).

Similarly to Proposition 19 one can then prove:

Proposition 33. Let $n \in \mathbb{N}$. Let $P$ be an $n$-graded poset. Let $i \in\{1,2, \ldots, n\}$. Let $\mathbb{K}$ be a field. Let $v \in \widehat{P}$. Let $f \in \mathbb{K}^{\widehat{P}}$.

(a) If $\operatorname{deg} v \neq i$, then $\left(R_{i} f\right)(v)=f(v)$.

(b) If $\operatorname{deg} v=i$, then

$$
\left(R_{i} f\right)(v)=\frac{1}{f(v)} \cdot \frac{\sum_{\substack{u \in \widehat{P} ; \\ u \ll v}} f(u)}{\sum_{\substack{u \in \widehat{P} ; \\ u>v}} \frac{1}{f(u)}} .
$$

Notice that using the proof of Proposition 33, it is easy to give an alternative proof of Corollary 29 (in the same way as we saw that an alternative proof of Corollary 16 could be given using the proofs of Propositions 19, 20 and 22).

\section{5 w-tuples}

This section continues the study of birational rowmotion on graded posets by introducing a "fingerprint" or "checksum" of a $\mathbb{K}$-labelling called the w-tuple, defined by summing ratios of elements between successive degrees (i.e., rows in the Hasse diagram). This wtuple serves to extract some information from a $\mathbb{K}$-labelling; we will later see how to make the "rest" of the labelling more manageable.

Definition 34. For $f \in \mathbb{K}^{\widehat{P}}$ and $i \in\{0,1, \ldots, n\}$, define $\mathbf{w}_{i}(f) \in \mathbb{K}$ by

$$
\mathbf{w}_{i}(f)=\sum_{\substack{x \in \widehat{P}_{i} ; y \in \widehat{P}_{i+1} ; \\ y \gg x}} \frac{f(x)}{f(y)} .
$$

(This element is not always defined, but is defined in the "generic" case when $0 \notin f(\widehat{P})$.) 
Intuitively, one could think of $\mathbf{w}_{i}(f)$ as a kind of "checksum" for the labelling $f$, displaying how much its labels at degree $i+1$ differ from those at degree $i$. Of course, in general, the knowledge of $\mathbf{w}_{i}(f)$ for all $i \in\{0,1, \ldots, n\}$ is far from sufficient to reconstruct the whole labelling $f$; however, in Definition 45, we will introduce the so-called homogenization of $f$, which will provide "complementary data" to these $\mathbf{w}_{i}(f)$. For now we show that the $\mathbf{w}_{i}(f)$ behave in a rather simple way under the maps $R$ and $R_{j}$.

Definition 35. For $f \in \mathbb{K}^{\widehat{P}}$, we call the $(n+1)$-tuple $\left(\mathbf{w}_{0}(f), \mathbf{w}_{1}(f), \ldots, \mathbf{w}_{n}(f)\right)$ the w-tuple of the $\mathbb{K}$-labelling $f$.

Proposition 36. For every $i \in\{1,2, \ldots, n\}$ and every $f \in \mathbb{K}^{\widehat{P}}$ we have

$$
\begin{aligned}
& \left(\mathbf{w}_{0}\left(R_{i} f\right), \mathbf{w}_{1}\left(R_{i} f\right), \ldots, \mathbf{w}_{n}\left(R_{i} f\right)\right) \\
& =\left(\mathbf{w}_{0}(f), \mathbf{w}_{1}(f), \ldots, \mathbf{w}_{i-2}(f), \mathbf{w}_{i}(f), \mathbf{w}_{i-1}(f), \mathbf{w}_{i+1}(f), \mathbf{w}_{i+2}(f), \ldots, \mathbf{w}_{n}(f)\right) .
\end{aligned}
$$

In other words, the map $R_{i}$ changes the w-tuple of a $\mathbb{K}$-labelling by interchanging its $(i-1)$-st entry with its $i$-th entry (where the entries are labelled starting at 0$)$.

The proof of this is a straightforward computation, taking cases $j=i, j=i-1$, and $j \notin\{i-1, i\}$. Details are left to the reader, or may be found in [GrRoArX].

From Proposition 36, and (5), we conclude:

Proposition 37. The map $R$ changes the w-tuple of any $f \in \mathbb{K}^{\widehat{P}}$ by shifting it cyclically: For every $f \in \mathbb{K}^{\widehat{P}}$, we have

$$
\left(\mathbf{w}_{0}(R f), \mathbf{w}_{1}(R f), \ldots, \mathbf{w}_{n}(R f)\right)=\left(\mathbf{w}_{n}(f), \mathbf{w}_{0}(f), \mathbf{w}_{1}(f), \ldots, \mathbf{w}_{n-1}(f)\right) .
$$

As a consequence of Proposition 37, the map $R^{n+1}$ (for an $n$-graded poset $P$ ) leaves the w-tuple of a $\mathbb{K}$-labelling fixed.

\section{Graded rescaling of labellings}

In general, birational rowmotion $R$ has something that one might call an "avalanche effect": If $f$ and $g$ are two $\mathbb{K}$-labellings of a poset $P$ which differ from each other only in their labels at one single element $v$, then the labellings $R f$ and $R g$ (in general) differ at all elements covering $v$ and all elements beneath $v$, and further applications of $R$ make the labellings even more different. Thus a change of just one label in a labelling will often "spread" through a large part of the poset when $R$ is repeatedly applied; the effect of such a change is hard to track in general. Thus, knowing the behavior of one particular $\mathbb{K}$-labelling $f$ under $R$ does not help us at understanding the behaviors of $\mathbb{K}$-labellings obtained from $f$ by changing labels at particular elements. However, if $P$ is a graded poset and we simultaneously multiply the labels at all elements of a given degree in a given labelling of $P$ with a given scalar, then the changes this causes to the behavior of the labelling under $R$ are rather predictable. We are going to formalize this observation in this section, proving some explicit formulas for how birational rowmotion $R$ and its 
iterates react to such rescalings. These explicit formulas will be subsumed into slick conclusions in Section 7, where we will introducing a notion of homogeneous equivalence which formalizes the idea of a "labelling modulo scalar factors at each degree".

The following definition formalizes the idea of multiplying the labels at all elements of a certain degree with one and the same scalar factor:

Definition 38. Let $P$ be an $n$-graded poset. Let $f \in \mathbb{K}^{\widehat{P}}$, and $\operatorname{let}^{9}\left(a_{0}, a_{1}, \ldots, a_{n+1}\right) \in$ $\left(\mathbb{K}^{\times}\right)^{n+2}$ be an $(n+2)$-tuple of nonzero elements of $\mathbb{K}$. We define a $\mathbb{K}$-labelling $\left(a_{0}, a_{1}, \ldots, a_{n+1}\right) b f \in \mathbb{K}^{\widehat{P}}$ by

$$
\left(\left(a_{0}, a_{1}, \ldots, a_{n+1}\right) b f\right)(v)=a_{\operatorname{deg} v} \cdot f(v) \quad \text { for every } v \in \widehat{P} .
$$

We preserve the notations and the setting of this definition for the rest of this section. Assuming that all applications of $R$ and $R_{i}$ are well-defined, we have the following straightforward consequences for $R_{i}, R$, and $R^{\ell}$.

Proposition 39. For every $i \in\{1,2, \ldots, n\}$, we have

$$
R_{i}\left(\left(a_{0}, a_{1}, \ldots, a_{n+1}\right) b f\right)=\left(a_{0}, a_{1}, \ldots, a_{i-1}, \frac{a_{i+1} a_{i-1}}{a_{i}}, a_{i+1}, a_{i+2}, \ldots, a_{n+1}\right) b\left(R_{i} f\right)
$$

Proposition 40. Let $r=\frac{a_{n+1}}{a_{n}}$. Then,

$$
R\left(\left(a_{0}, a_{1}, \ldots, a_{n+1}\right) b f\right)=\left(a_{0}, r a_{0}, r a_{1}, \ldots, r a_{n-1}, a_{n+1}\right) b(R f),
$$

Proof of Proposition 40. We claim that every $j \in\{1,2, \ldots, n+1\}$ satisfies

$$
\begin{aligned}
& \left(R_{j} \circ R_{j+1} \circ \ldots \circ R_{n}\right)\left(\left(a_{0}, a_{1}, \ldots, a_{n+1}\right) b f\right) \\
& =\left(a_{0}, a_{1}, a_{2}, \ldots, a_{j-1}, r a_{j-1}, r a_{j}, \ldots, r a_{n-1}, a_{n+1}\right) b\left(\left(R_{j} \circ R_{j+1} \circ \ldots \circ R_{n}\right) f\right) .
\end{aligned}
$$

Indeed, (7) is easily verified by reverse induction over $j$ (that is, induction over $n+1-j$ ), using Proposition 39 in the step. Now, applying (7) to $j=1$ and recalling that $R=$ $R_{1} \circ R_{2} \circ \ldots \circ R_{n}$, we obtain Proposition 40 .

Proposition 41. For every $k, \ell \in\{0,1, \ldots, n+1\}$, define an element $\widehat{a}_{k}^{(\ell)} \in \mathbb{K}^{\times}$by

$$
\widehat{a}_{k}^{(\ell)}=\left\{\begin{array}{ll}
\frac{a_{n+1} a_{k-\ell}}{a_{n+1-\ell}}, & \text { if } k \geqslant \ell ; \\
\frac{a_{n+1+k-\ell} a_{0}}{a_{n+1-\ell}}, & \text { if } k<\ell
\end{array} .\right.
$$

Then every $\ell \in\{0,1, \ldots, n+1\}$ satisfies

$$
R^{\ell}\left(\left(a_{0}, a_{1}, \ldots, a_{n+1}\right) b f\right)=\left(\widehat{a}_{0}^{(\ell)}, \widehat{a}_{1}^{(\ell)}, \ldots, \widehat{a}_{n+1}^{(\ell)}\right) b\left(R^{\ell} f\right)
$$

\footnotetext{
${ }^{9}$ Here and in the following, $\mathbb{K}^{\times}$denotes the multiplicative group of nonzero elements of $\mathbb{K}$.
} 
Proof of Proposition 41. This proof is a completely straightforward induction over $\ell$, with the base case being trivial and the induction step relying on Proposition 40. To simplify the computations, notice that $\widehat{a}_{k}^{(\ell)}=\frac{a_{n+1+k-\ell} a_{0}}{a_{n+1-\ell}}$ if $k \leqslant \ell$.

As a corollary, rescaling labellings commutes with $R^{n+1}$ for an $n$-graded poset $P$ :

Corollary 42. We have $R^{n+1}\left(\left(a_{0}, a_{1}, \ldots, a_{n+1}\right) b f\right)=\left(a_{0}, a_{1}, \ldots, a_{n+1}\right) b\left(R^{n+1} f\right)$.

Finally, straightforward computations from the definitions show how rescaling a labeling by degree affects w-tuples:

Proposition 43. The w-tuple of the $\mathbb{K}$-labelling $\left(a_{0}, a_{1}, \ldots, a_{n+1}\right) b f$ is

$$
\left(\frac{a_{0}}{a_{1}} \mathbf{w}_{0}(f), \frac{a_{1}}{a_{2}} \mathbf{w}_{1}(f), \ldots, \frac{a_{n}}{a_{n+1}} \mathbf{w}_{n}(f)\right) .
$$

\section{Homogeneous labellings}

In the previous section, we have quantified how the rescaling of all labels at a given degree affects a labelling (of a graded poset) under birational rowmotion. In this section, we will introduce a notion of "homogeneous labellings" which (roughly speaking) are "labellings up to rescaling at a given degree" in the same way as a point in a projective space can be regarded as "a point in the affine space up to rescaling the coordinates". To be precise, we will need to restrict ourselves to considering only "zero-free" labellings (a Zariski-dense open subset of all labellings) for the same reason as we need to exclude 0 when defining a projective space. Once done with the definitions, we will see that birational rowmotion (and the maps $R_{i}$ ) can be defined on homogeneous labellings (making use of the results of the previous section).

Definition 44. (a) For every $\mathbb{K}$-vector space $V$, let $\mathbb{P}(V)$ denote the projective space of $V$ (that is, the set $V \backslash\{0\}$ modulo proportionality).

(b) For every $n \in \mathbb{N}$, we let $\mathbb{P}^{n}(\mathbb{K})$ denote the projective space $\mathbb{P}\left(\mathbb{K}^{n+1}\right)$.

Definition 45. (a) Denote by $\overline{\mathbb{K}^{\widehat{P}}}$ the product $\prod_{i=1}^{n} \mathbb{P}\left(\mathbb{K}^{\widehat{P}_{i}}\right)$ of projective spaces. Notice that the product is just a Cartesian product of algebraic varieties, and a reader unfamiliar with algebraic geometry can just regard it as a Cartesian product of sets. ${ }^{10}$

We have $\overline{\mathbb{K}^{\widehat{P}}}=\prod_{i=1}^{n} \mathbb{P}\left(\mathbb{K}^{\widehat{P}_{i}}\right) \cong \prod_{i=1}^{n} \mathbb{P}^{\left|\widehat{P}_{i}\right|-1}(\mathbb{K})$ (since every $i \in\{1,2, \ldots, n\}$ satisfies $\left.\mathbb{P}\left(\mathbb{K}^{\widehat{P}_{i}}\right) \cong \mathbb{P}^{\left|\widehat{P}_{i}\right|-1}(\mathbb{K})\right)$. We denote the elements of $\overline{\mathbb{K}^{\widehat{P}}}$ as homogeneous labellings.

Notice that $\overline{\mathbb{K}^{\widehat{P}}}=\prod_{i=1}^{n} \mathbb{P}\left(\mathbb{K}^{\widehat{P}_{i}}\right) \cong \prod_{i=0}^{n+1} \mathbb{P}\left(\mathbb{K}^{\widehat{P}_{i}}\right)$ (as algebraic varieties). This is because $\mathbb{K}^{\widehat{P}_{0}}$ and $\mathbb{K}^{\widehat{P}_{n+1}}$ are 1 -dimensional vector spaces (since $\left|\widehat{P}_{0}\right|=1$ and $\left|\widehat{P}_{n+1}\right|=1$ ), and thus the projective spaces $\mathbb{P}\left(\mathbb{K}^{\widehat{P}_{0}}\right)$ and $\mathbb{P}\left(\mathbb{K}^{\widehat{P}_{n+1}}\right)$ each consist of a single point. 
(b) A $\mathbb{K}$-labelling $f \in \mathbb{K}^{\widehat{P}}$ is said to be zero-free if for every $i \in\{0,1, \ldots, n+1\}$, there exists some $v \in \widehat{P}_{i}$ satisfying $f(v) \neq 0$ (i.e., there exists no $i \in\{0,1, \ldots, n+1\}$ such that $f$ is identically 0 on all elements of $\widehat{P}$ having degree $i$.) Let $\mathbb{K}_{\neq 0}^{\widehat{P}}$ be the set of all zero-free $\mathbb{K}$-labellings. Clearly, this set $\mathbb{K}_{\neq 0}^{\widehat{P}}$ is a Zariski-dense open subset of $\mathbb{K}^{\widehat{P}}$.

(c) Identify the set $\mathbb{K}^{\widehat{P}}$ with $\prod_{i=0}^{n+1} \mathbb{K}^{\widehat{P}_{i}}$ in the obvious way (since, as a set, $\widehat{P}=\bigsqcup_{i=0}^{n+1} \widehat{P}_{i}$ ). Using the identifications $\mathbb{K}^{\widehat{P}} \cong \prod_{i=0}^{n+1} \mathbb{K}^{\widehat{P}_{i}}$ and $\overline{\mathbb{K}^{\widehat{P}}} \cong \prod_{i=0}^{n+1} \mathbb{P}\left(\mathbb{K}^{\widehat{P}_{i}}\right)$, we now define a rational map $\pi: \mathbb{K}^{\widehat{P}} \rightarrow \overline{\mathbb{K}^{\widehat{P}}}$ as the product of the canonical projections $\mathbb{K}^{\widehat{P}_{i}} \rightarrow \mathbb{P}\left(\mathbb{K}^{\widehat{P}_{i}}\right)$ (which are defined everywhere outside of the $\{0\}$ subsets) over all $i \in\{0,1, \ldots, n+1\}$. Notice that the domain of definition of this rational map $\pi$ is precisely $\mathbb{K}_{\neq 0}^{\widehat{P}}$. For every $f \in \mathbb{K}^{\widehat{P}}$, we denote $\pi(f)$ as the homogenization of the $\mathbb{K}$-labelling $f$.

(d) Two zero-free $\mathbb{K}$-labellings $f \in \mathbb{K}^{\widehat{P}}$ and $g \in \mathbb{K}^{\widehat{P}}$ are said to be homogeneously equivalent if and only if they satisfy one of the following equivalent conditions:

Condition 1: For every $i \in\{0,1, \ldots, n+1\}$ and any two elements $x$ and $y$ of $\widehat{P}_{i}$, we have $\frac{f(x)}{f(y)}=\frac{g(x)}{g(y)}$.

Condition 2: There exists an $(n+2)$-tuple $\left(a_{0}, a_{1}, \ldots, a_{n+1}\right) \in\left(\mathbb{K}^{\times}\right)^{n+2}$ such that $g=\left(a_{0}, a_{1}, \ldots, a_{n+1}\right) b f$ (that is, such that every $x \in \widehat{P}$ satisfies $g(x)=a_{\operatorname{deg} x} \cdot f(x)$ ).

Condition 3: We have $\pi(f)=\pi(g)$.

(The equivalence between these three conditions is very easy to check. We will never actually use Condition 1.)

Remark 46. Clearly, homogeneous equivalence is an equivalence relation on the set $\mathbb{K}_{\neq 0}^{\widehat{P}}$ of all zero-free $\mathbb{K}$-labellings. We can identify $\overline{\mathbb{K}^{\widehat{P}}}$ with the quotient of the set $\mathbb{K}_{\neq 0}^{\widehat{P}}$ modulo this relation. Then, $\pi$ becomes the canonical projection map $\mathbb{K}^{\widehat{P}} \rightarrow \overline{\mathbb{K}^{\widehat{P}}}$.

Being zero-free is a very weak condition on a $\mathbb{K}$-labelling (indeed the zero-free $\mathbb{K}$ labellings form a Zariski-dense open subset of the space of all $\mathbb{K}$-labellings), and the $\mathbb{K}$ labellings which don't satisfy this condition are rather useless for us (if $f$ is a $\mathbb{K}$-labelling which is not zero-free, then $R^{2} f$ is not well-defined, and usually not even $R f$ is welldefined). We are almost never giving up any generality if we require a labelling to be zero-free.

Remark 47. Let $f \in \mathbb{K}_{\neq 0}^{\widehat{P}}$ and $\left(a_{0}, a_{1}, \ldots, a_{n+1}\right) \in\left(\mathbb{K}^{\times}\right)^{n+2}$. Then $\left(a_{0}, a_{1}, \ldots, a_{n+1}\right) b f$ is also zero-free. (This follows immediately from the definitions.)

\footnotetext{
${ }^{10}$ The structure of an algebraic variety will only be needed to define the Zariski topology on $\overline{\mathbb{K}^{\widehat{P}}}$, which is more or less obvious already (e.g., when we say that something holds "for almost every element $x$ of $\prod_{i=1}^{n} \mathbb{P}\left(\mathbb{K}^{\widehat{P}_{i}}\right)$ ", we could equivalently say that it holds "for $x=\operatorname{proj}(X)$ for almost every element $X$ of $\prod_{i=1}^{n}\left(\mathbb{K}^{\widehat{P}_{i}} \backslash\{0\}\right) "$, where proj is the canonical map $\prod_{i=1}^{n}\left(\mathbb{K}^{\widehat{P}_{i}} \backslash\{0\}\right) \rightarrow \prod_{i=1}^{n} \mathbb{P}\left(\mathbb{K}^{\widehat{P}_{i}}\right)$ defined as the product of the projections $\left.\mathbb{K}^{\widehat{P}_{i}} \backslash\{0\} \rightarrow \mathbb{P}\left(\mathbb{K}^{\widehat{P}_{i}}\right)\right)$.
} 
Definition 48. For every zero-free $f \in \mathbb{K}^{\widehat{P}}$ and every $i \in\{1,2, \ldots, n\}$, the image of the restriction of $f: \widehat{P} \rightarrow \mathbb{K}$ to $\widehat{P}_{i}$ under the canonical projection $\mathbb{K}^{\widehat{P}_{i}} \rightarrow \mathbb{P}\left(\mathbb{K}^{\widehat{P}_{i}}\right)$ will be denoted by $\pi_{i}(f)$. This image $\pi_{i}(f)$ encodes the values of $f$ at the elements of $\widehat{P}$ of degree $i$ up to multiplying all these values by a common nonzero scalar. Notice that

$$
\pi(f)=\left(\pi_{1}(f), \pi_{2}(f), \ldots, \pi_{n}(f)\right) .
$$

We next note that birational rowmotion preserves homogeneous equivalence.

Corollary 49. If $f, g \in \mathbb{K}^{\widehat{P}}$ are two homogeneously equivalent zero-free $\mathbb{K}$-labellings, then $R_{i} f$ is homogeneously equivalent to $R_{i} g$ (as long as $R_{i} f$ and $R_{i} g$ are zero-free).

Corollary 50. If $f, g \in \mathbb{K}^{\widehat{P}}$ are two homogeneously equivalent zero-free $\mathbb{K}$-labellings, then $R f$ is homogeneously equivalent to $R g$ (as long as $R f$ and $R g$ are zero-free).

Notice that Corollary 49 would not be valid if we were to replace $R_{i}$ by a single toggle $T_{v}$ ! So the operators $R_{i}$ in some sense combine the nice properties of $T_{v}$ (like being an involution, cf. Proposition 32) with the nice properties of $R$ (like having an easily describable action on w-tuples, cf. Proposition 36, and respecting homogeneous equivalence, cf. Corollary 49).

Proof of Corollary 49. By Condition 2 in Definition 45 (d), there exists an $(n+2)$-tuple $\left(a_{0}, a_{1}, \ldots, a_{n+1}\right) \in\left(\mathbb{K}^{\times}\right)^{n+2}$ such that $g=\left(a_{0}, a_{1}, \ldots, a_{n+1}\right) b f$. Thus,

$$
R_{i} g=R_{i}\left(\left(a_{0}, a_{1}, \ldots, a_{n+1}\right) b f\right)=\left(a_{0}, a_{1}, \ldots, a_{i-1}, \frac{a_{i+1} a_{i-1}}{a_{i}}, a_{i+1}, a_{i+2}, \ldots, a_{n+1}\right) b\left(R_{i} f\right)
$$

(by Proposition 39). Thus, $R_{i} f$ and $R_{i} g$ are homogenously equivalent.

Proof of Corollary 50. Corollary 49 shows that the map $R_{i}$ (for every $1 \leqslant i \leqslant n$ ) preserves homogeneous equivalence of $\mathbb{K}$-labellings. Hence, so does the composition $R_{1} \circ R_{2} \circ \ldots \circ R_{n}$ of these maps, which is $R$ (by Proposition 31 ).

Let us introduce a general piece of notation:

Definition 51. Let $S$ and $T$ be two sets, equipped with respective equivalence relations $\sim_{S}$ and $\sim_{T}$. Let $\bar{S}=S / \sim_{S}$ and $\bar{T}=T / \sim_{T}$ be the respective quotients, and $\pi_{S}: S \rightarrow \bar{S}$ and $\pi_{T}: T \rightarrow \bar{T}$ the canonical projections. Let $f: S \rightarrow T$ be a map. If $\bar{f}: \bar{S} \rightarrow \bar{T}$ is a map for which the diagram

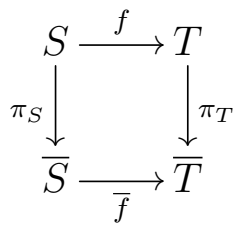

is commutative, then we say that "the map $f$ descends to the map $\bar{f}$ ". It is easy to see that there exists at most one map $\bar{f}: \bar{S} \rightarrow \bar{T}$ such that the map $f$ descends to the map 
$\bar{f}$ (for given $S, T, \sim_{S}, \sim_{T}$ and $f$ ). Moreover, the existence of a map $\bar{f}: \bar{S} \rightarrow \bar{T}$ such that the map $f$ descends to the map $\bar{f}$ is equivalent to the statement that every two elements $x$ and $y$ of $S$ satisfying $x \sim_{S} y$ satisfy $f(x) \sim_{T} f(y)$.

The above statements are not literally true if we replace the map $f: S \rightarrow T$ by a partial map $f: S \rightarrow T$. However, when $S$ and $T$ are two algebraic varieties and $\sim_{S}$ and $\sim_{T}$ are algebraic equivalences (i.e., equivalence relations defined by polynomial relations between coordinates of points) and $f: S \rightarrow T$ is a rational map, then the above statements still are true (of course, with $\bar{f}$ being a partial map).

Definition 52. Let $i \in\{1,2, \ldots, n\}$. Because of Corollary 49, the rational map $R_{i}$ : $\mathbb{K}^{\widehat{P}} \rightarrow \mathbb{K}^{\widehat{P}}$ descends (through the projection $\pi: \mathbb{K}^{\widehat{P}} \rightarrow \overline{\mathbb{K}^{\widehat{P}}}$ ) to a partial map $\overline{\mathbb{K}^{\widehat{P}}} \rightarrow \overline{\mathbb{K}^{\widehat{P}}}$, which we denote by $\overline{R_{i}}$. Thus, the diagram

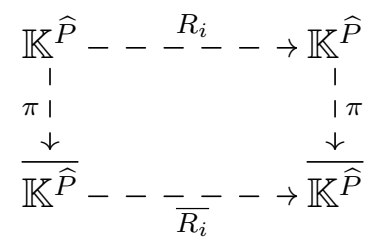

is commutative.

Definition 53. Define the partial map $\bar{R}: \overline{\mathbb{K}^{\widehat{P}}} \rightarrow \overline{\mathbb{K}^{\widehat{P}}}$ by $\bar{R}=\overline{R_{1}} \circ \overline{R_{2}} \circ \ldots \circ \overline{R_{n}}$. Then, the diagram

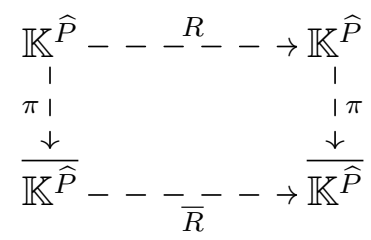

is commutative ${ }^{11}$. In other words, $\bar{R}$ is the partial map $\overline{\mathbb{K}^{\widehat{P}}} \rightarrow \overline{\mathbb{K}^{\widehat{P}}}$ to which the partial map $R: \mathbb{K}^{\widehat{P}} \rightarrow \mathbb{K}^{\widehat{P}}$ descends (through the projection $\pi: \mathbb{K}^{\widehat{P}} \rightarrow \overline{\mathbb{K}^{\widehat{P}}}$ ).

The next result says roughly that a zero-free $\mathbb{K}$-labelling $f \in \mathbb{K}^{\widehat{P}}$ is almost always uniquely determined by three specifications: its w-tuple $\left(\mathbf{w}_{0}(f), \mathbf{w}_{1}(f), \ldots, \mathbf{w}_{n}(f)\right)$, its homogenization $\pi(f)$ and the value $f(0)$. More precisely:

Proposition 54. Let $f, g \in \mathbb{K}_{\neq 0}^{\widehat{P}}$ satisfy

- $\pi(f)=\pi(g)$;

- $f(0)=g(0) ;$ and

- $\left(\mathbf{w}_{0}(f), \mathbf{w}_{1}(f), \ldots, \mathbf{w}_{n}(f)\right)=\left(\mathbf{w}_{0}(g), \mathbf{w}_{1}(g), \ldots, \mathbf{w}_{n}(g)\right) \in\left(\mathbb{K}^{\times}\right)^{n+1}$.

\footnotetext{
${ }^{11}$ Proof. We have $R=R_{1} \circ R_{2} \circ \ldots \circ R_{n}$ and $\bar{R}=\overline{R_{1}} \circ \overline{R_{2}} \circ \ldots \circ \overline{R_{n}}$. Hence, the diagram (10) can be obtained by stringing together the diagrams (9) for all $i \in\{1,2, \ldots, n\}$ and then removing the "interior edges".
} 
Then, $f=g$.

Proposition 54 is easily proven by reconstructing $f$ and $g$ "bottom-up" along $\widehat{P}$. Alternatively, we can use Proposition 43, as follows:

Proof of Proposition 54. Since $\pi(f)=\pi(g)$, we know that $f$ and $g$ are homogeneously equivalent. By Condition 2 in Definition $45(\mathrm{~d})$, this means that there exists an $(n+2)$ tuple $\left(a_{0}, a_{1}, \ldots, a_{n+1}\right) \in\left(\mathbb{K}^{\times}\right)^{n+2}$ such that $g=\left(a_{0}, a_{1}, \ldots, a_{n+1}\right) b f$; hence,

$$
\text { (the w-tuple of } g)=\left(\frac{a_{0}}{a_{1}} \mathbf{w}_{0}(f), \frac{a_{1}}{a_{2}} \mathbf{w}_{1}(f), \ldots, \frac{a_{n}}{a_{n+1}} \mathbf{w}_{n}(f)\right)
$$

(by Proposition 43). Compared with

(the w-tuple of $g)=\left(\mathbf{w}_{0}(g), \mathbf{w}_{1}(g), \ldots, \mathbf{w}_{n}(g)\right)=\left(\mathbf{w}_{0}(f), \mathbf{w}_{1}(f), \ldots, \mathbf{w}_{n}(f)\right)$, this yields

$$
\left(\frac{a_{0}}{a_{1}} \mathbf{w}_{0}(f), \frac{a_{1}}{a_{2}} \mathbf{w}_{1}(f), \ldots, \frac{a_{n}}{a_{n+1}} \mathbf{w}_{n}(f)\right)=\left(\mathbf{w}_{0}(f), \mathbf{w}_{1}(f), \ldots, \mathbf{w}_{n}(f)\right) .
$$

Hence, $\frac{a_{i}}{a_{i+1}}=1$ for every $i \in\{0,1, \ldots, n\}$. (Here we use the assumption that $\left(\mathbf{w}_{0}(f), \mathbf{w}_{1}(f), \ldots, \mathbf{w}_{n}(f)\right) \in\left(\mathbb{K}^{\times}\right)^{n+1}$.) This forces $a_{0}=a_{1}=\ldots=a_{n+1}$, so the labels of $g$ are obtained from those of $f$ by multiplying by one single constant. The assumption $f(0)=g(0)$ shows that this constant is 1 , and thus $f=g$.

Definition 55. In the following, if $S$ is a finite set, and $q$ is an element of a projective space $\mathbb{P}\left(\mathbb{K}^{S}\right)$ of the free vector space with basis $S$, and $k$ is an integer, then $q^{k}$ will denote the element of $\mathbb{P}\left(\mathbb{K}^{S}\right)$ obtained by replacing every homogeneous coordinate of $q$ by its $k$-th power. This is well-defined (and will mostly be used for $k=-1$ ). In particular, this definition applies to $S=\{1,2, \ldots, n\}$ (in which case $\mathbb{K}^{S}=\mathbb{K}^{n}$ ).

We can explicitly describe the action of the $\overline{R_{i}}$ when the "structure of the poset $P$ between degrees $i-1, i$ and $i+1$ " is particularly simple:

Proposition 56. Fix $i \in\{1,2, \ldots, n\}$, and assume that every $u \in \widehat{P}_{i}$ and every $v \in \widehat{P}_{i+1}$ satisfy $u \lessdot v$. Assume further that every $u \in \widehat{P}_{i-1}$ and every $v \in \widehat{P}_{i}$ satisfy $u \lessdot v$. Then,

$$
\begin{aligned}
& \left(\pi_{1}\left(R_{i} f\right), \pi_{2}\left(R_{i} f\right), \ldots, \pi_{n}\left(R_{i} f\right)\right) \\
& =\left(\pi_{1}(f), \pi_{2}(f), \ldots, \pi_{i-1}(f),\left(\pi_{i}(f)\right)^{-1}, \pi_{i+1}(f), \pi_{i+2}(f), \ldots, \pi_{n}(f)\right) .
\end{aligned}
$$

From this proposition, we obtain two corollaries:

Corollary 57. Fix $i \in\{1,2, \ldots, n\}$. Assume that every $u \in \widehat{P}_{i}$ and every $v \in \widehat{P}_{i+1}$ satisfy $u \lessdot v$. Assume further that every $u \in \widehat{P}_{i-1}$ and every $v \in \widehat{P}_{i}$ satisfy $u \lessdot v$. Let $\widetilde{f}=\left(\widetilde{f}_{1}, \widetilde{f}_{2}, \ldots, \widetilde{f}_{n}\right) \in \overline{\mathbb{K}^{\widehat{P}}}$. Then,

$$
\overline{R_{i}}(\tilde{f})=\left(\tilde{f}_{1}, \tilde{f}_{2}, \ldots, \tilde{f}_{i-1}, \tilde{f}_{i}^{-1}, \widetilde{f}_{i+1}, \widetilde{f}_{i+2}, \ldots, \tilde{f}_{n}\right) .
$$


Corollary 58. Assume that, for every $i \in\{1,2, \ldots, n-1\}$, every $u \in \widehat{P}_{i}$ and every $v \in \widehat{P}_{i+1}$ satisfy $u \lessdot v$. Let $f \in \mathbb{K}^{\widehat{P}}$ be zero-free. Then,

$$
\left(\pi_{1}(R f), \pi_{2}(R f), \ldots, \pi_{n}(R f)\right)=\left(\left(\pi_{1}(f)\right)^{-1},\left(\pi_{2}(f)\right)^{-1}, \ldots,\left(\pi_{n}(f)\right)^{-1}\right) .
$$

\section{Order}

In this short section, we will relate the orders of the maps $R$ and $\bar{R}$ for a graded poset $P$. The relation will later be used to better understand both of these orders. We begin by defining the order of a partial map.

Definition 59. Let $S$ be a set.

(a) If $\alpha$ and $\beta$ are two partial maps from the set $S$, then we write " $\alpha=\beta$ " if and only if every $s \in S$ for which both $\alpha(s)$ and $\beta(s)$ are well-defined satisfies $\alpha(s)=\beta(s)$. This is not a well-behaved notation per se, e.g., it is possible that three partial maps $\alpha$, $\beta$ and $\gamma$ satisfy $\alpha=\beta$ and $\beta=\gamma$ but not $\alpha=\gamma$. However, we will only use this notation for rational maps and their quotients (and, of course, total maps); in all of these cases, the notation is well-behaved (e.g., if $\alpha, \beta$ and $\gamma$ are three rational maps satisfying $\alpha=\beta$ and $\beta=\gamma$, then $\alpha=\gamma$, because the intersection of two Zariski-dense open subsets is Zariski-dense and open).

(b) The order of a partial map $\varphi: S \rightarrow S$ is defined to be the smallest positive integer $k$ satisfying $\varphi^{k}=\mathrm{id}_{S}$, if such a positive integer $k$ exists, and $\infty$ otherwise. Here, we are disregarding the fact that $\varphi$ is only a partial map; we will be working only with dominant rational maps and their quotients (and total maps), so nothing will go wrong.

We denote the order of a partial map $\varphi: S \rightarrow S$ as ord $\varphi$.

Convention 60. In the following, we are going to occasionally make arithmetical statements involving the symbol $\infty$. We declare that 0 and $\infty$ are divisible by $\infty$, but no positive integer is divisible by $\infty$. We further declare that every positive integer (but not 0$)$ divides $\infty$. We set $\operatorname{lcm}(a, \infty)$ and $\operatorname{lcm}(\infty, a)$ to mean $\infty$ whenever $a$ is a positive integer.

As a consequence of Proposition 54, we have:

Proposition 61. Let $n \in \mathbb{N}$. Let $\mathbb{K}$ be a field. Let $P$ be an n-graded poset. Then, ord $R=\operatorname{lcm}(n+1$, ord $\bar{R})$. (Recall that $\operatorname{lcm}(n+1, \infty)$ is to be understood as $\infty$.)

Proof of Proposition 61. The proof of this boils down to considering the effect of $R$ on the w-tuple $\left(\mathbf{w}_{0}(f), \mathbf{w}_{1}(f), \ldots, \mathbf{w}_{n}(f)\right)$ and on the homogenization $\pi(f)$ of a $\mathbb{K}$-labelling $f$. The former is a cyclic shift (by Proposition 37), with order $n+1$, and the latter is $\bar{R}$. It is now easy to see (invoking Proposition 54) that the order of $R$ is the $1 \mathrm{~cm}$ of the orders of these two actions. We outline the steps here; full details may be found in [GrRoArX].

1st step: Use commutativity of the diagram (10) to show $\bar{R}^{\ell} \circ \pi=\pi \circ R^{\ell}$ for all $\ell \in \mathbb{N}$. Thus, $\bar{R}^{\ell}=$ id whenever $R^{\ell}=\mathrm{id}$, so ord $\bar{R} \mid \operatorname{ord} R$. 
2nd step: Assume ord $\bar{R}=m \neq \infty$ (otherwise the statement is easy), and let $\ell=$ $\operatorname{lcm}(n+1, m)$. To show that $R^{\ell}=\mathrm{id}$, it suffices to show that $R^{\ell} f=f$ for almost every (Zariski) $f \in \mathbb{K}_{\neq 0}^{\widehat{P}}$. A simple argument shows that almost every $f \in \mathbb{K}^{\widehat{P}}$ is zero-free and has (well-defined) nonzero $\mathbf{w}_{i}(f)$ for each $i$. By Proposition 37, the labellings $R^{\ell} f$ and $f$ have the same w-tuple (since $n+1 \mid \ell$ ), and from ord $\bar{R}=m \mid \ell$ we obtain $\pi\left(R^{\ell} f\right)=\pi(f)$. So using Corollary 21 and applying Proposition 54 to $g=R^{\ell} f$, we get $R^{\ell} f=f$. Thus, $R^{\ell}=\mathrm{id}$, so ord $R \mid \ell$.

3rd step: To show the inverse divisibility $(\ell \mid$ ord $R$ ), first assume ord $R=q \neq \infty$ (else the claim is obvious). It is easy to see that for almost every zero-free $\mathbb{K}$-labelling $f$ of $P$, the entries of the w-tuple $\left(\mathbf{w}_{0}(f), \mathbf{w}_{1}(f), \ldots, \mathbf{w}_{n}(f)\right)$ of $f$ are pairwise distinct. So there exists $f \in \mathbb{K}_{\neq 0}^{\widehat{P}}$ with this property such that $R^{k} f$ is well-defined for all $k \in\{0,1, \ldots, q\}$. Then $R^{q} f=f$, so they have the same w-tuple. But by pairwise-distinctness, this can only happen if $n+1 \mid q$. Combined with the first step, this gives $\ell \mid \operatorname{ord} R$.

\section{The opposite poset}

Before we move on to the first interesting class of posets for which we can compute the order of birational rowmotion, let us prove an easy "symmetry property" of birational rowmotion. For this section, we only assume that $P$ is a finite poset (not necessarily graded).

Definition 62. Let $P$ be a poset. Then, $P^{\text {op }}$ will denote the poset defined on the same ground set as $P$ but with the order relation defined by

$$
\left(\left(a<_{P_{\mathrm{op}}} b \text { if and only if } b<_{P} a\right) \text { for all } a \in P \text { and } b \in P\right)
$$

The poset $P^{\mathrm{op}}$ is called the opposite poset (or dual poset [Stan11]) of $P$.

Definition 63. We denote the maps $R$ and $\bar{R}$ by $R_{P}$ and $\bar{R}_{P}$, respectively, so as to make their dependence on $P$ explicit.

We can now state a symmetry property of ord $R$ (as defined in Definition 59):

Proposition 64. We have ord $\left(R_{P_{\text {op }}}\right)=\operatorname{ord}\left(R_{P}\right)$ and ord $\left(\bar{R}_{P_{\text {op }}}\right)=\operatorname{ord}\left(\bar{R}_{P}\right)$.

Proof of Proposition 64. Define a rational map $\kappa: \mathbb{K}^{\widehat{P}} \rightarrow \mathbb{K}^{\widehat{P \text { op }}}$ by

$$
(\kappa f)(w)=\left\{\begin{array}{ll}
\frac{1}{f(w)}, & \text { if } w \in P ; \\
\frac{1}{f(1)}, & \text { if } w=0 ; \\
\frac{1}{f(0)}, & \text { if } w=1
\end{array} \quad \text { for every } w \in \widehat{P^{\text {op }}} \text { for every } f \in \mathbb{K}^{\widehat{P}} .\right.
$$

This map $\kappa$ is a birational map. (Its inverse map is defined in the same way.)

We claim that $\kappa \circ R_{P}=R_{P \text { op }}^{-1} \circ \kappa$. 
Indeed, it is easy to see (by computation) that every element $v \in P$ satisfies

$$
\kappa \circ T_{v}=T_{v} \circ \kappa
$$

where the $T_{v}$ on the left hand side is defined with respect to the poset $P$, and the $T_{v}$ on the right hand side is defined with respect to the poset $P^{\mathrm{op}}$. Now, let $\left(v_{1}, v_{2}, \ldots, v_{m}\right)$ be a linear extension of $P$. Then, $\left(v_{m}, v_{m-1}, \ldots, v_{1}\right)$ is a linear extension of $P^{\text {op }}$, so that Proposition 23 (applied to $P^{\mathrm{op}}$ and $\left(v_{m}, v_{m-1}, \ldots, v_{1}\right)$ instead of $P$ and $\left(v_{1}, v_{2}, \ldots, v_{m}\right)$ ) yields that $R_{P^{\text {op }}}^{-1}=T_{v_{1}} \circ T_{v_{2}} \circ \ldots \circ T_{v_{m}}: \widehat{\mathbb{K}^{\text {op }}} \rightarrow \widehat{\mathbb{K}^{\text {oop }}}$. On the other hand, the definition of $R_{P}$ yields $R_{P}=T_{v_{1}} \circ T_{v_{2}} \circ \ldots \circ T_{v_{m}}: \mathbb{K}^{\widehat{P}} \rightarrow \mathbb{K}^{\widehat{P}}$. Now, using (11), it is easy to see that

$$
\kappa \circ\left(T_{v_{1}} \circ T_{v_{2}} \circ \ldots \circ T_{v_{m}}\right)=\left(T_{v_{1}} \circ T_{v_{2}} \circ \ldots \circ T_{v_{m}}\right) \circ \kappa .
$$

Since the $T_{v_{1}} \circ T_{v_{2}} \circ \ldots \circ T_{v_{m}}$ on the left hand side equals $R_{P}$, and the $T_{v_{1}} \circ T_{v_{2}} \circ \ldots \circ T_{v_{m}}$ on the right hand side equals $R_{P \text { op }}^{-1}$, this rewrites as $\kappa \circ R_{P}=R_{P \text { op }}^{-1} \circ \kappa$. Since $\kappa$ is a birational map, this shows that $R_{P}$ and $R_{P \text { op }}^{-1}$ are birationally equivalent, so that $\operatorname{ord}\left(R_{P}\right)=\operatorname{ord}\left(R_{P \text { op }}^{-1}\right)=$ ord $\left(R_{P \text { op }}\right)$. Since $\kappa$ commutes with homogenization, we also obtain the birational equivalence of the maps $\bar{R}_{P}$ and $\bar{R}_{P^{\text {op }}}^{-1}$, whence ord $\left(\bar{R}_{P}\right)=\operatorname{ord}\left(\bar{R}_{P^{\text {op }}}^{-1}\right)=\operatorname{ord}\left(\bar{R}_{P_{\text {op }}}\right)$.

\section{Skeletal posets}

We will now introduce a class of posets which we call "skeletal posets". Roughly speaking, these are graded posets built up inductively from the empty poset by the operations of (1) disjoint union of two $n$-graded posets (with the same $n$ ) and (2) "grafting" on an antichain (generalizing the idea of grafting a tree on a new root). In particular, this class includes all graded forests (oriented either away from the roots or towards the roots) as well as various other posets.

Definition 65. Let $P$ and $Q$ be two $n$-graded posets. We denote by $P Q$ the disjoint union of the posets $P$ and $Q$ (denoted by $P+Q$ in [Stan11, §3.2]). Its poset structure is defined in such a way that any element of $P$ and any element of $Q$ are incomparable, while $P$ and $Q$ are subposets of $P Q$. Clearly, $P Q$ is also an $n$-graded poset.

Definition 66. Let $P$ be an $n$-graded poset. Let $k$ be a positive integer. We denote by $B_{k} P$ the result of adding $k$ new elements to the poset $P$, and declaring these $k$ elements to be smaller than each of the elements of $P$ (but incomparable with each other). Clearly, $B_{k} P$ is an $(n+1)$-graded poset.

Definition 67. Let $n \in \mathbb{N}$. Let $P$ be an $n$-graded poset. Let $k$ be a positive integer. We denote by $B_{k}^{\prime} P$ the result of adding $k$ new elements to the poset $P$, and declaring these $k$ elements to be larger than each of the elements of $P$ (but incomparable with each other). Clearly, $B_{k}^{\prime} P$ is an $(n+1)$-graded poset.

In the notation of Stanley ([Stan11, §3.2]), $B_{k} P=A_{k} \oplus P$ and $B_{k}^{\prime} P=P \oplus A_{k}$, where $A_{k}$ denotes the $k$-element antichain. It is easy to see that $B_{k} P$ and $B_{k}^{\prime} P$ are "symmetric" notions with respect to taking the opposite poset (Def. 62): 
Proposition 68. Let $P$ be an n-graded poset. Then, $B_{k}^{\prime} P=\left(B_{k}\left(P^{\mathrm{op}}\right)\right)^{\mathrm{op}}$.

Definition 69. We define the class of skeletal posets inductively by means of the following axioms:

- The empty poset is skeletal.

- If $P$ is an $n$-graded skeletal poset and $k$ is a positive integer, then the posets $B_{k} P$ and $B_{k}^{\prime} P$ are skeletal.

- If $n$ is a nonnegative integer and $P$ and $Q$ are two $n$-graded skeletal posets, then the poset $P Q$ is skeletal.

Notice that every skeletal poset is graded, and that every graded rooted forest (made into a poset by having every node smaller than its children) is a skeletal poset. (Recall that our sense of "graded" means all maximal chains have the same length, so not all trees are graded.) Indeed, every graded rooted forest can be constructed from $\varnothing$ using merely the operations $P \mapsto B_{1} P$ and $(P, Q) \mapsto P Q$. Also, every graded rooted arborescence (i.e., the opposite poset of a graded rooted tree) is a skeletal poset (for a similar reason).

Example 70. The rooted forest $\bullet-\bullet \bullet \bullet$ is skeletal, and in fact can be

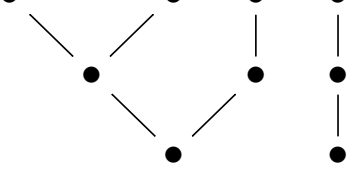

written as $\left(B_{1}\left(\left(B_{1}\left(B_{2} \varnothing\right)\right)\left(B_{1}\left(B_{1} \varnothing\right)\right)\right)\right)\left(B_{1}\left(B_{1}\left(B_{1} \varnothing\right)\right)\right)$. (This form of writing is not unique, since $B_{2} \varnothing=\left(B_{1} \varnothing\right)\left(B_{1} \varnothing\right)$.)

The tree $\quad$ can be written as $B_{1}\left(\left(B_{1} \varnothing\right)\left(B_{1}\left(B_{1} \varnothing\right)\right)\right)$, but is not skeletal

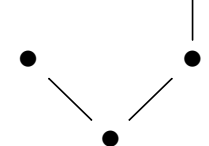

because $B_{1} \varnothing$ and $B_{1}\left(B_{1} \varnothing\right)$ are not $n$-graded with one and the same $n$.

The poset

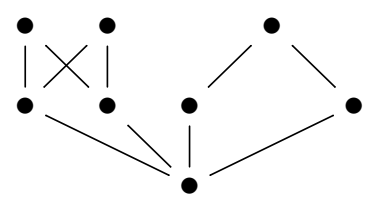

is neither a tree nor an arborescence, but it has the

form $B_{1}\left(\left(B_{2}\left(B_{2} \varnothing\right)\right)\left(B_{1}^{\prime}\left(B_{2} \varnothing\right)\right)\right)$ and is skeletal.

Our main result on skeletal posets is the following:

Proposition 71. If $P$ is a skeletal poset, then ord $\left(R_{P}\right)$ and ord $\left(\bar{R}_{P}\right)$ are finite.

We first build up some machinery for determining ord $\left(R_{P}\right)$ and ord $\left(\bar{R}_{P}\right)$ given such orders in smaller posets. Here is a very basic fact to get started:

Proposition 72. Fix $n \in \mathbb{N}$, and let $P$ and $Q$ be two n-graded posets. Then, we have $\operatorname{ord}\left(R_{P Q}\right)=\operatorname{lcm}\left(\operatorname{ord}\left(R_{P}\right)\right.$, ord $\left.\left(R_{Q}\right)\right)$.

Proof of Proposition 72. The proof of this is as easy as it looks: a $\mathbb{K}$-labelling of the disjoint union $P Q$ can be regarded as a pair of a $\mathbb{K}$-labelling of $P$ and a $\mathbb{K}$-labelling of $Q$ (with identical labels at 0 and 1 ), and the map $R$ (as well as all $R_{i}$ ) acts on these labellings independently. 
The analogue of Proposition 72 with all $R$ 's replaced by $\bar{R}$ 's is false. Instead, ord $\left(\bar{R}_{P Q}\right)$ can be computed as follows:

Proposition 73. Fix $n \in \mathbb{N}$, and let $P$ and $Q$ be two $n$-graded posets. Let $\mathbb{K}$ be a field. Then, ord $\left(\bar{R}_{P Q}\right)=\operatorname{lcm}\left(\operatorname{ord}\left(R_{P}\right)\right.$, ord $\left.\left(R_{Q}\right)\right)$. (As we know, this is equal to ord $\left(R_{P Q}\right)$.)

The most useful point here is the statement that ord $\left(\bar{R}_{P Q}\right) \mid \operatorname{lcm}\left(\operatorname{ord}\left(R_{P}\right)\right.$, ord $\left.\left(R_{Q}\right)\right)$. This follows immediately from Propositions 61 and 73. The reverse divisibility is mainly a technical result which can be proved by splitting a labelling of $P Q$ into a labelling of $P$ and one of $Q$ and comparing how $\bar{R}$ affects these labellings. We refer to [GrRoArX, §9] for details.

Now, let us track the effect of $B_{k}$ on the order of $\bar{R}$ :

Proposition 74. Let $P$ be an n-graded poset.

(a) We have ord $\left(\bar{R}_{B_{1} P}\right)=\operatorname{ord}\left(\bar{R}_{P}\right)$.

(b) For every integer $k>1$, we have ord $\left(\bar{R}_{B_{k} P}\right)=\operatorname{lcm}\left(2\right.$, ord $\left.\left(\bar{R}_{P}\right)\right)$.

Proof of Proposition 74. We only need to prove that

$$
\operatorname{ord}\left(\bar{R}_{B_{k} P}\right)=\left\{\begin{array}{l}
\operatorname{lcm}\left(2, \text { ord }\left(\bar{R}_{P}\right)\right), \quad \text { if } k>1 ; \\
\operatorname{ord}\left(\bar{R}_{P}\right), \quad \text { if } k=1
\end{array} .\right.
$$

Let us make some conventions:

- For any $n$-tuple $\left(\alpha_{1}, \alpha_{2}, \ldots, \alpha_{n}\right)$ and any object $\beta$, let $\beta \curlywedge \alpha$ denote the $(n+1)$-tuple $\left(\beta, \alpha_{1}, \alpha_{2}, \ldots, \alpha_{n}\right)$.

- We are going to identify $P$ with a subposet of $B_{k} P$ in the obvious way. However, the degree maps of these posets differ and have to be distinguished. We also embed $\widehat{P}$ into $\widehat{B_{k} P}$ by identifying the respective 0 s and the respective $1 \mathrm{~s}$.

- The rational maps $\pi: \mathbb{K}^{\widehat{P}} \rightarrow \overline{\mathbb{K}^{\widehat{P}}}$ and $\pi: \widehat{\mathbb{K}^{B_{k} P}} \rightarrow \overline{\mathbb{K}^{\widehat{k_{k} P}}}$ are denoted by the same letter and can be distinguished by their different domains. We will also use the letter $\pi$ to denote the rational map $\mathbb{K}^{k} \rightarrow \mathbb{P}\left(\mathbb{K}^{k}\right)$ obtained from the canonical projection $\mathbb{K}^{k} \backslash\{0\} \rightarrow \mathbb{P}\left(\mathbb{K}^{k}\right)$ of the nonzero vectors in $\mathbb{K}^{k}$ onto the projective space.

Now the operation $B_{k}$ clearly raises the degree of every element of $P$ by 1 , while the $k$ newly added elements all obtain degree 1 in $B_{k} P$. Formally speaking, this means that $\widehat{B}_{k} P_{i}=\widehat{P}_{i-1}$ for every $i \in\{2,3, \ldots, n+1\}$, while ${\widehat{B_{k} P}}_{1}$ is a $k$-element set. Moreover, for any $i \in\{2,3, \ldots, n+1\}$, any $u \in \widehat{B} k_{i}=\widehat{P}_{i-1}$ and any $v \in \widehat{B}_{k} P_{i+1}=\widehat{P}_{i}$, we have

$$
u \lessdot v \text { in } \widehat{B_{k} P} \text { if and only if } u \lessdot v \text { in } \widehat{P} \text {. }
$$


We have $\widehat{\mathbb{K}^{B_{k} P_{i}}}=\mathbb{K}^{\widehat{P}_{i-1}}$ for every $i \in\{2,3, \ldots, n+1\}$, whereas $\widehat{\mathbb{K}^{\hat{B}_{k} P_{1}}} \cong \mathbb{K}^{k}$. We will actually identify $\mathbb{K}^{\overline{B k}_{k}}$ with $\mathbb{K}^{k}$. Now,

$$
\begin{aligned}
\overline{\mathbb{K}^{\widehat{B_{k} P}}} & =\prod_{i=1}^{n+1} \mathbb{P}\left(\mathbb{K}^{\widehat{B_{k} P_{i}}}\right)=\mathbb{P}(\underbrace{\widehat{\mathbb{K}^{B_{k} P_{1}}}}_{=\mathbb{K}^{k}}) \times \prod_{i=2}^{n+1} \mathbb{P}(\underbrace{\widehat{\mathbb{K}^{B_{k} P_{i}}}}_{=\mathbb{K}^{\widehat{P}_{i-1}}}) \\
& =\mathbb{P}\left(\mathbb{K}^{k}\right) \times \prod_{i=2}^{n+1} \mathbb{P}\left(\mathbb{K}^{\widehat{P}_{i-1}}\right)=\mathbb{P}\left(\mathbb{K}^{k}\right) \times \underbrace{\prod_{i=1}^{n} \mathbb{P}\left(\mathbb{K}^{\widehat{P}_{i}}\right)}_{=\widehat{\mathbb{K}^{\widehat{P}}}}=\mathbb{P}\left(\mathbb{K}^{k}\right) \times \overline{\mathbb{K}^{\widehat{P}}} .
\end{aligned}
$$

Thus, the elements of $\overline{\mathbb{K}^{\widehat{k_{k} P}}}$ have the form $\widetilde{p} \wedge \widetilde{g}$, where $\widetilde{p} \in \mathbb{P}\left(\mathbb{K}^{k}\right)$ and $\widetilde{g} \in \overline{\mathbb{K}^{\widehat{P}}}$.

On the other hand, $\widehat{P}$ is the subposet $\widehat{B_{k} P} \backslash \widehat{B_{k} P}$ of $\widehat{B_{k} P}$. Thus, we can define a map $\Phi: \mathbb{K}^{k} \times \mathbb{K}^{\widehat{P}} \rightarrow \widehat{K^{B_{k} P}}$ by setting

$$
(\Phi(p, g))(v)=\left\{\begin{array}{cl}
p(v), & \text { if } v \in \widehat{B_{k} P} ; \\
g(v), & \text { if } v \notin \widehat{B_{k} P_{1}}
\end{array} \quad \text { for every } v \in \widehat{B_{k} P}\right.
$$

for every $(p, g) \in \mathbb{K}^{k} \times \mathbb{K}^{\widehat{P}}$. Here, the term $p(v)$ is to be understood by means of regarding $p$ as an element of $\mathbb{K}^{\widehat{B_{k} P_{1}}}$ ( since $p \in \mathbb{K}^{k}=\mathbb{K}^{B_{k} P_{1}}$ ). Clearly, $\Phi$ is a bijection. Moreover, it is easy to see that

$$
\pi(\Phi(p, g))=\pi(p) \wedge \pi(g) \quad \text { for all } p \in \mathbb{K}^{k} \text { and } g \in \mathbb{K}^{\widehat{P}}
$$

(where the $\pi$ on the left hand side is the map $\pi: \widehat{\mathbb{K}^{\hat{k}_{k} P}} \rightarrow \widehat{\mathbb{K}^{\widehat{k_{k} P}}}$, whereas the $\pi$ in " $\pi(p)$ " is the map $\pi: \mathbb{K}^{k} \rightarrow \mathbb{P}\left(\mathbb{K}^{k}\right)$, and the $\pi$ in " $\pi(g)$ " is the map $\left.\pi: \mathbb{K}^{\widehat{P}} \rightarrow \overline{\mathbb{K}^{\widehat{P}}}\right)$.

Now, we claim that every $\widetilde{p} \in \mathbb{P}\left(\mathbb{K}^{k}\right)$ and $\widetilde{g} \in \overline{\mathbb{K}^{\widehat{P}}}$ satisfy

$$
\left(\overline{R_{i}}\right)_{B_{k} P}(\widetilde{p} \wedge \widetilde{g})=\widetilde{p} \wedge\left(\overline{R_{i-1}}\right)_{P}(\widetilde{g}) \quad \text { for all } i \in\{2,3, \ldots, n+1\}
$$

and

$$
\left(\overline{R_{1}}\right)_{B_{k} P}(\widetilde{p} \wedge \widetilde{g})=\widetilde{p}^{-1}<\widetilde{g} .
$$

Proof of (15) and (16): In order to prove (15), it is clearly enough to show that every $p \in \mathbb{K}^{k}$ and $g \in \mathbb{K}^{\widehat{P}}$ satisfy

$$
\left(R_{i}\right)_{B_{k} P}(p \wedge g) \sim p \curlywedge\left(R_{i-1}\right)_{P}(g) \quad \text { for all } i \in\{2,3, \ldots, n+1\},
$$

where the sign $\sim$ stands for homogeneous equivalence.

It is easy to prove the relation (17) for $i>2$ (because if $i>2$, then the elements of $\widehat{B_{k} P}$ having degrees $i-1, i$ and $i+1$ are precisely the elements of $\widehat{P}$ having degrees $i-2$, $i-1$ and $i$, and therefore toggling the elements of ${\widehat{B_{k} P}}_{i}$ in $p \wedge g$ has precisely the same effect as toggling the elements of $\widehat{P}_{i-1}$ in $g$ while leaving $p$ fixed, so that we even get the 
stronger assertion that $\left.\left(R_{i}\right)_{B_{k} P}(p \wedge g)=p \curlywedge\left(R_{i-1}\right)_{P}(g)\right)$. It is not much harder to check that it also holds for $i=2$ (indeed, for $i=2$, the only difference between toggling the elements of $\widehat{B}_{k} P_{i}$ in $p \wedge g$ and toggling the elements of $\widehat{P}_{i-1}$ in $g$ while leaving $p$ fixed is a scalar factor which is identical across all elements being toggled in either poset ${ }^{12}$; therefore, the results are the same up to homogeneous equivalence).

Finally, (16) is trivial to check (e.g., using Corollary 57).

But recall that $\bar{R}=\overline{R_{1}} \circ \overline{R_{2}} \circ \ldots \circ \overline{R_{n}}$ for any $n$-graded poset. Hence, $\bar{R}_{B_{k} P}=$ $\left(\overline{R_{1}}\right)_{B_{k} P} \circ\left(\overline{R_{2}}\right)_{B_{k} P} \circ\left(\overline{R_{3}}\right)_{B_{k} P} \circ \ldots \circ\left(\overline{R_{n+1}}\right)_{B_{k} P}$ (because $B_{k} P$ is an $(n+1)$-graded poset) and $\bar{R}_{P}=\left(\overline{R_{1}}\right)_{P} \circ\left(\overline{R_{2}}\right)_{P} \circ \ldots \circ\left(\overline{R_{n}}\right)_{P}$ (because $P$ is an $n$-graded poset). Because of these equalities, and by (15) and (16), it is now easy to see that every $\widetilde{p} \in \mathbb{P}\left(\mathbb{K}^{k}\right)$ and $\widetilde{g} \in \overline{\mathbb{K}^{\widehat{P}}}$ satisfy

$$
\bar{R}_{B_{k} P}(\widetilde{p} \curlywedge \widetilde{g})=\widetilde{p}^{-1} \curlywedge \bar{R}_{P}(\widetilde{g}) .
$$

Furthermore, every $\widetilde{p} \in \mathbb{P}\left(\mathbb{K}^{k}\right)$ and $\widetilde{g} \in \overline{\mathbb{K}^{\widehat{P}}}$ satisfy

$$
\bar{R}_{B_{k} P}^{\ell}(\widetilde{p} \wedge \widetilde{g})=\widetilde{p}^{(-1)^{\ell}}<\bar{R}_{P}^{\ell}(\widetilde{g}) \quad \text { for all } \ell \in \mathbb{N} .
$$

(This is proven by induction over $\ell$, using (18).)

We know that the elements of $\overline{\mathbb{K}^{\widehat{B_{k}}}}$ have the form $\widetilde{p} \wedge \widetilde{g}$, where $\widetilde{p} \in \mathbb{P}\left(\mathbb{K}^{k}\right)$ and $\widetilde{g} \in \overline{\mathbb{K}^{\widehat{P}}}$. Conversely, every element $\widetilde{p} \wedge \widetilde{g}$ with $\tilde{p} \in \mathbb{P}\left(\mathbb{K}^{k}\right)$ and $\widetilde{g} \in \overline{\mathbb{K}^{\widehat{P}}}$ lies in $\overline{\mathbb{K}^{B_{k} P}}$. Hence, for every $\ell \in \mathbb{N}$, we have the following equivalence of assertions:

$$
\begin{aligned}
& \left(\bar{R}_{B_{k} P}^{\ell}=\mathrm{id}\right) \Longleftrightarrow\left(\text { every } \tilde{p} \in \mathbb{P}\left(\mathbb{K}^{k}\right) \text { and } \widetilde{g} \in \overline{\mathbb{K}^{\widehat{P}}} \text { satisfy } \bar{R}_{B_{k} P}^{\ell}(\widetilde{p} \wedge \widetilde{g})=\widetilde{p} \wedge \widetilde{g}\right) \\
& \Longleftrightarrow\left(\text { every } \widetilde{p} \in \mathbb{P}\left(\mathbb{K}^{k}\right) \text { and } \widetilde{g} \in \overline{\mathbb{K}^{\widehat{P}}} \text { satisfy } \widetilde{p}^{(-1)^{\ell}} \wedge \bar{R}_{P}^{\ell}(\widetilde{g})=\widetilde{p} \wedge \widetilde{g}\right) \quad(\text { by }(19)) \\
& \Longleftrightarrow\left(\text { every } \widetilde{p} \in \mathbb{P}\left(\mathbb{K}^{k}\right) \text { and } \widetilde{g} \in \overline{\mathbb{K}^{\widehat{P}}} \text { satisfy } \widetilde{p}^{(-1)^{\ell}}=\widetilde{p} \text { and } \bar{R}_{P}^{\ell}(\widetilde{g})=\widetilde{g}\right) \\
& \Longleftrightarrow(\underbrace{\text { every } \widetilde{p} \in \mathbb{P}\left(\mathbb{K}^{k}\right) \text { satisfies } \widetilde{p}^{(-1)^{\ell}}=\widetilde{p}}_{\text {this is equivalent to }(2 \mid \ell \text { if } k>1)}, \text { and } \underbrace{\text { every } \widetilde{g} \in \overline{\mathbb{K}^{\widehat{P}}} \text { satisfies } \bar{R}_{P}^{\ell}(\widetilde{g})=\widetilde{g}}_{\text {this is equivalent to ord }\left(\bar{R}_{P}\right) \mid \ell}) \\
& \Longleftrightarrow\left(\left\{\begin{array}{l}
\operatorname{lcm}\left(2, \text { ord }\left(\bar{R}_{P}\right)\right), \quad \text { if } k>1 \\
\left.\operatorname{ord}\left(\bar{R}_{P}\right), \quad \mid \ell\right) .
\end{array}\right.\right.
\end{aligned}
$$

Hence, for every $\ell \in \mathbb{N}$, we have the following equivalence of assertions:

$$
\begin{aligned}
& \left(\operatorname{ord}\left(\bar{R}_{B_{k} P}\right) \mid \ell\right) \Longleftrightarrow\left(\bar{R}_{B_{k} P}^{\ell}=\mathrm{id}\right) \Longleftrightarrow\left(\left\{\begin{array}{l}
\left.\operatorname{lcm}\left(2, \operatorname{ord}\left(\bar{R}_{P}\right)\right), \quad \text { if } k>1 ; \quad \mid \ell\right) . \\
\operatorname{ord}\left(\bar{R}_{P}\right), \quad \text { if } k=1
\end{array}\right.\right. \\
& \text { Consequently, ord }\left(\bar{R}_{B_{k} P}\right)=\left\{\begin{array}{l}
\operatorname{lcm}\left(2, \text { ord }\left(\bar{R}_{P}\right)\right), \quad \text { if } k>1 ; \\
\operatorname{ord}\left(\bar{R}_{P}\right), \quad
\end{array}\right.
\end{aligned}
$$

\footnotetext{
${ }^{12}$ because every $u \in{\widehat{B_{k} P}}_{1}$ and every $v \in \widehat{B}_{k P}$ satisfy $u \lessdot v$
} 
Here is an analogue of Proposition 74:

Proposition 75. Let $P$ be an n-graded poset.

(a) We have ord $\left(\bar{R}_{B_{1}^{\prime} P}\right)=$ ord $\left(\bar{R}_{P}\right)$.

(b) For every integer $k>1$, we have ord $\left(\bar{R}_{B_{k}^{\prime} P}\right)=\operatorname{lcm}\left(2\right.$, ord $\left.\left(\bar{R}_{P}\right)\right)$.

The proof of this is very similar (though not exactly identical) to that of Proposition 74 . Alternatively, it is easy to deduce Proposition 75 from Proposition 74 using Proposition 64 and Proposition 68.

Proof of Proposition 71. For any skeletal poset $T$, we can compute ord $\left(R_{T}\right)$ and ord $\left(\bar{R}_{T}\right)$ inductively using Propositions $72-75$ (and the fact that ord $\left(R_{\varnothing}\right)=1$ and ord $\left(\bar{R}_{\varnothing}\right)=1$ ). More precisely:

- If $T$ is the empty poset $\varnothing$, then $\operatorname{ord}\left(R_{T}\right)=\operatorname{ord}\left(R_{\varnothing}\right)=1=\operatorname{ord}\left(\bar{R}_{T}\right)=\operatorname{ord}\left(\bar{R}_{\varnothing}\right)$.

- If $T$ has the form $B_{k} P$ for some $n$-graded skeletal poset $P$ and some positive integer $k$, then Proposition 74 yields

$$
\operatorname{ord}\left(\bar{R}_{T}\right)=\operatorname{ord}\left(\bar{R}_{B_{k} P}\right)=\left\{\begin{array}{l}
\operatorname{lcm}\left(2, \operatorname{ord}\left(\bar{R}_{P}\right)\right), \quad \text { if } k>1 ; \\
\operatorname{ord}\left(\bar{R}_{P}\right), \quad \text { if } k=1
\end{array},\right.
$$

and Proposition 61 yields ord $\left(R_{T}\right)=\operatorname{lcm}\left(n+1\right.$, ord $\left.\left(\bar{R}_{T}\right)\right)$.

- Analogously one can compute ord $\left(R_{T}\right)$ and ord $\left(\bar{R}_{T}\right)$ if $T$ has the form $B_{k}^{\prime} P$.

- If $T$ has the form $P Q$ for two WLOG nonempty $n$-graded skeletal posets $P$ and $Q$, then Proposition 72 yields ord $\left(R_{P Q}\right)=\operatorname{lcm}\left(\operatorname{ord}\left(R_{P}\right)\right.$, ord $\left.\left(R_{Q}\right)\right)$, and Proposition 73 yields ord $\left(\bar{R}_{P Q}\right)=\operatorname{lcm}\left(\operatorname{ord}\left(R_{P}\right)\right.$, ord $\left.\left(R_{Q}\right)\right)$.

This gives an algorithm for inductively computing ord $\left(R_{T}\right)$ and ord $\left(\bar{R}_{T}\right)$ for a skeletal poset $T$. It is clear that these orders as computed by this algorithms will be finite.

As the proof of Proposition 71 shows, we can recursively compute (rather than just bound from the above) the orders of $R_{P}$ and $\bar{R}_{P}$ for any skeletal poset $P$ without doing any computations in $\mathbb{K}$. (This also shows that the orders of $R_{P}$ and $\bar{R}_{P}$ don't depend on the base field $\mathbb{K}$ as long as $\mathbb{K}$ is infinite and $P$ is skeletal.)

In the case of forests and trees we obtain a concrete bound:

Corollary 76. Let $P$ be an n-graded poset which is also a rooted forest (made into a poset by having every node smaller than its children).

(a) Then, ord $\left(R_{P}\right) \mid \operatorname{lcm}(1,2, \ldots, n+1)$.

(b) Moreover, if $P$ is a tree, then ord $\left(\bar{R}_{P}\right) \mid \operatorname{lcm}(1,2, \ldots, n)$.

Corollary 76 is also valid if we replace "every node smaller than its children" by "every node larger than its children", and the proof is exactly analogous.

Proof of Corollary 76. Use strong induction on $|P|$, applying Propositions 74 (a) and 61 when $P$ is a tree, and Proposition 72 when $P$ is a forest. (See [GrRoArX] for details.) 


\section{Postscript: Classical rowmotion on skeletal posets}

The above results concerning birational rowmotion on skeletal posets suggest the question of what can be said about classical rowmotion (on the set of order ideals) on this class of posets. Indeed, while the classical rowmotion map (as opposed to the birational one) has been the object of several studies (e.g., [StWi11] and [CaFl95]), it seems that this rather simple case has never been explicitly covered. Let us bridge this gap and derive the counterparts of Propositions 74 and 71 and Corollary 76 for classical rowmotion.

To start we define (classical) toggles on order ideals (an analogue of Definition 11). Recall that an order ideal of $P$ means a subset $S$ of $P$ such that every $s \in S$ and $p \in P$ with $p \leqslant s$ satisfy $p \in S$. We denote the set of all order ideals of $P$ by $J(P)$.

Definition 77. Let $P$ be a finite poset. Let $v \in P$. Define a map $\mathbf{t}_{v}: J(P) \rightarrow J(P)$ by

$$
\mathbf{t}_{v}(S)=\left\{\begin{array}{l}
S \cup\{v\}, \text { if } v \notin S \text { and } S \cup\{v\} \in J(P) ; \\
S \backslash\{v\}, \text { if } v \in S \text { and } S \backslash\{v\} \in J(P) ; \\
S, \text { otherwise }
\end{array} \quad \text { for every } S \in J(P) .\right.
$$

(This is clearly well-defined.) This map $\mathbf{t}_{v}$ will be called the classical $v$-toggle.

We can rewrite this definition in more "local" terms, by replacing the conditions " $S \cup\{v\} \in J(P)$ " and " $S \backslash\{v\} \in J(P)$ " by the respectively equivalent conditions "every element $u \in P$ satisfying $u \lessdot v$ lies in $S$ " and "no element $u \in P$ satisfying $u \gg v$ lies in $S$ " (in fact, the equivalence of these conditions is easily seen). Hence, we obtain the following analogue to our definition of $T_{v}$ :

Proposition 78. Let $P$ be a finite poset. Let $v \in P$. For every $S \in J(P)$, we have:

(a) If $w$ is an element of $P$ such that $w \neq v$, then we have $w \in \mathbf{t}_{v}(S)$ if and only if $w \in S$.

(b) We have $v \in \mathbf{t}_{v}(S)$ if and only if

$$
\begin{aligned}
& (v \in S \text { and not }(\text { no element } u \in P \text { satisfying } u \gg v \text { lies in } S)) \\
& \text { or }(v \notin S \text { and (every element } u \in P \text { satisfying } u \lessdot v \text { lies in } S)) .
\end{aligned}
$$

While the complicated logical statement in Proposition 78 (b) can be simplified, the form we have stated it in exhibits its similarity to our definition of $T_{v}$ particularly well. This, in fact, is more than a similarity: If we allow $\mathbb{K}$ to be a semifield rather than a field, we can regard the classical $v$-toggle $\mathbf{t}_{v}$ as a restriction of the birational toggle $T_{v}$ (when $\mathbb{K}$ is chosen appropriately) ${ }^{13}$. Hence, some theorems about birational toggles can

\footnotetext{
${ }^{13}$ Here are the details: Let Trop $\mathbb{Z}$ be the tropical semiring over $\mathbb{Z}$, that is, the semiring obtained by endowing the set $\mathbb{Z} \cup\{-\infty\}$ with the binary operation $(a, b) \mapsto \max \{a, b\}$ as "addition" and the binary operation $(a, b) \mapsto a+b$ as "multiplication" (where the usual rules for sums involving $-\infty$ apply). Then, Trop $\mathbb{Z}$ is a semifield, with $(a, b) \mapsto a-b$ serving as "subtraction", with $-\infty$ serving as "zero" and with the integer 0 serving as "one". Now, to every order ideal $S \in J(P)$, we can assign a (Trop $\mathbb{Z}$ )-labelling
} 
be used to derive analogous theorems about classical toggles ${ }^{14}$. We will not use this tactic in the following, because often it will be easier to study the classical $v$-toggles on their own. However, many of the properties of classical toggles (and classical rowmotion) that we are going to discuss will have proofs that are parallel to the proofs of the analogous results about birational toggles. We will omit these proofs when the analogy is glaring enough.

We have the following easily-verified analogues of Proposition 12, Proposition 14 and Corollary 16:

Proposition 79. Let $P$ be a finite poset. Let $v \in P$. Then, the map $\mathbf{t}_{v}$ is an involution on $J(P)$ (that is, we have $\mathbf{t}_{v}^{2}=\mathrm{id}$ ).

Proposition 80. Let $P$ be a finite poset. Let $v \in P$ and $w \in P$. Then, $\mathbf{t}_{v} \circ \mathbf{t}_{w}=\mathbf{t}_{w} \circ \mathbf{t}_{v}$, unless we have either $v \lessdot w$ or $w \lessdot v$.

Corollary 81. Let $P$ be a finite poset. Let $\left(v_{1}, v_{2}, \ldots, v_{m}\right)$ be a linear extension of $P$. Then, the map $\mathbf{t}_{v_{1}} \circ \mathbf{t}_{v_{2}} \circ \ldots \circ \mathbf{t}_{v_{m}}: J(P) \rightarrow J(P)$ is well-defined and independent of the choice of the linear extension $\left(v_{1}, v_{2}, \ldots, v_{m}\right)$.

The three results above are observations made on [CaFl95, page 546] (in somewhat different notation).

Two convenient advantages of the classical setup are that we don't have to worry about denominators becoming zero, so our maps are actual maps rather than partial maps, and that we don't have to pass to the poset $\widehat{P}$. We can now define rowmotion in analogy to Definition 17:

Definition 82. Let $P$ be a finite poset. Classical rowmotion (simply called "rowmotion" in existing literature) is defined as the map $\mathbf{r}:=\mathbf{t}_{v_{1}} \circ \mathbf{t}_{v_{2}} \circ \ldots \circ \mathbf{t}_{v_{m}}: J(P) \rightarrow J(P)$, where $\left(v_{1}, v_{2}, \ldots, v_{m}\right)$ is a linear extension of $P$. This map is well-defined by Corollary 81 .

To highlight the similarities between the classical and birational cases, let us state the analogue of Proposition 19:

Proposition 83. Let $P$ be a finite poset. Let $v \in P$. Let $S \in J(P)$. Then, $v \in \mathbf{r}(S)$ holds if and only if the following two conditions hold:

Condition 1: Every $u \in P$ satisfying $u \lessdot v$ belongs to $S$.

Condition 2: Either $v \notin S$, or there exists an $u \in \mathbf{r}(S)$ satisfying $u>v$. (The "either/or" is meant non-exclusively.)

tlab $S \in(\operatorname{Trop} \mathbb{Z})^{\widehat{P}}$, defined by

$$
(\operatorname{tlab} S)(v)=\left\{\begin{array}{c}
1, \text { if } v \notin S \cup\{0\} \\
0, \text { if } v \in S \cup\{0\}
\end{array}\right.
$$

This yields a map tlab $: J(P) \rightarrow(\text { Trop } \mathbb{Z})^{\widehat{P}}$, obviously injective. This map tlab satisfies $T_{v}$ otlab $=$ tlab $\circ \mathbf{t}_{v}$ for every $v \in P$. This allows us to regard the classical toggles $\mathbf{t}_{v}$ as restrictions of the birational toggles $T_{v}$, if we consider this map tlab as an inclusion. This reasoning goes back to Einstein and Propp [EiPr13].

${ }^{14}$ For example, we could derive Proposition 80 from Proposition 14 using this tactic. However, we could not derive (say) Proposition 101 from Proposition 71 this way, because the order of a restriction of a permutation could be a proper divisor of the order of the permutation. 
This proposition is easily seen to be equivalent to the following well-known equivalent description of rowmotion ([CaFl95, Lemma 1], translated into our notation):

Proposition 84. Let $P$ be a finite poset. Let $S \in J(P)$. Then, the maximal elements of $\mathbf{r}(S)$ are precisely the minimal elements of $P \backslash S$.

We record the analogue of Proposition 22:

Proposition 85. Let $P$ be a finite poset. Let $S$ and $T$ be two order ideals of $P$. Assume that for every $v \in P$, the relation $v \in T$ holds if and only if Conditions 1 and 2 of Proposition 83 hold with $\mathbf{r}(S)$ replaced by $T$. Then, $T=\mathbf{r}(S)$.

In analogy to Proposition 23, we have:

Proposition 86. Let $P$ be a finite poset. Then, classical rowmotion $\mathbf{r}$ is invertible. Its inverse $\mathbf{r}^{-1}$ is $\mathbf{t}_{v_{m}} \circ \mathbf{t}_{v_{m-1}} \circ \ldots \circ \mathbf{t}_{v_{1}}: J(P) \rightarrow J(P)$, where $\left(v_{1}, v_{2}, \ldots, v_{m}\right)$ is a linear extension of $P$.

We can study graded posets again. In analogy to Corollary 29, Definition 30, Proposition 31 and Proposition 32, we have:

Corollary 87. Let $P$ be an n-graded poset. Fix $i \in\{1,2, \ldots, n\}$. Let $\left(u_{1}, u_{2}, \ldots, u_{k}\right)$ be any list of the elements of $\widehat{P}_{i}$ with every element of $\widehat{P}_{i}$ appearing exactly once in the list. 15 Then, the map $\mathbf{r}_{i}:=\mathbf{t}_{u_{1}} \circ \mathbf{t}_{u_{2}} \circ \ldots \circ \mathbf{t}_{u_{k}}: J(P) \rightarrow J(P)$ is well-defined and independent of the choice of the list $\left(u_{1}, u_{2}, \ldots, u_{k}\right)$.

Proposition 88. Let $P$ be an n-graded poset. Then, $\mathbf{r}=\mathbf{r}_{1} \circ \mathbf{r}_{2} \circ \ldots \circ \mathbf{r}_{n}$.

Proposition 89. Let $P$ be an n-graded poset. Let $i \in\{1,2, \ldots, n\}$. Then, $\mathbf{r}_{i}$ is an involution on $J(P)$ (that is, $\mathbf{r}_{i}^{2}=\mathrm{id}$ ).

A parody of w-tuples can also be defined. The following is analogous to Definition 34:

Definition 90. For $S \in J(P)$ and $i \in\{1,2, \ldots, n\}$, set

$$
\mathbf{w}_{i}(S)=\left\{\begin{array}{l}
1, \text { if } P_{i} \subseteq S \text { and } P_{i+1} \cap S=\varnothing . \\
0, \text { otherwise }
\end{array} .\right.
$$

Here, $P_{j}$ denotes the subset $\operatorname{deg}^{-1}(\{j\})$ of $P$, with $P_{0}=P_{n+1}=\varnothing$.

Analogues of Proposition 36 and Proposition 37 are easily found:

Proposition 91. For any $S \in J(P)$ and $i \in\{1,2, \ldots, n\}$, we have

$$
\begin{aligned}
& \left(\mathbf{w}_{0}\left(\mathbf{r}_{i}(S)\right), \mathbf{w}_{1}\left(\mathbf{r}_{i}(S)\right), \ldots, \mathbf{w}_{n}\left(\mathbf{r}_{i}(S)\right)\right) \\
& =\left(\mathbf{w}_{0}(S), \mathbf{w}_{1}(S), \ldots, \mathbf{w}_{i-2}(S), \mathbf{w}_{i}(S), \mathbf{w}_{i-1}(S), \mathbf{w}_{i+1}(S), \mathbf{w}_{i+2}(S), \ldots, \mathbf{w}_{n}(S)\right) .
\end{aligned}
$$

\footnotetext{
${ }^{15}$ Note that $\widehat{P}_{i}$ is simply $\{v \in P \mid \operatorname{deg} v=i\}$, because $i$ equals neither 0 nor $n+1$. We are using the notation $\widehat{P}_{i}$ despite not working with $\widehat{P}$ merely to stress some analogies.
} 
Proposition 92. For any $S \in J(P)$, we have

$$
\left(\mathbf{w}_{0}(\mathbf{r}(S)), \mathbf{w}_{1}(\mathbf{r}(S)), \ldots, \mathbf{w}_{n}(\mathbf{r}(S))\right)=\left(\mathbf{w}_{n}(S), \mathbf{w}_{0}(S), \mathbf{w}_{1}(S), \ldots, \mathbf{w}_{n-1}(S)\right) .
$$

However, the $(n+1)$-tuple $\left(\mathbf{w}_{0}(S), \mathbf{w}_{1}(S), \ldots, \mathbf{w}_{n}(S)\right)$ obtained from an order ideal $S$ is not particularly informative. In fact, it is $(0,0, \ldots, 0)$ for "most" order ideals; here is what this means precisely:

Definition 93. An order ideal of $P$ is said to be level if and only if it has the form $P_{1} \cup P_{2} \cup \ldots \cup P_{i}$ for some $i \in\{0,1, \ldots, n\}$.

Easy properties of level order ideals are:

Proposition 94. Let $P$ be an n-graded poset.

(a) There exist precisely $n+1$ level order ideals of $P$, and those form an orbit under classical rowmotion $\mathbf{r}$. Namely, one has

$$
\mathbf{r}\left(P_{1} \cup P_{2} \cup \ldots \cup P_{i}\right)=\left\{\begin{array}{ll}
P_{1} \cup P_{2} \cup \ldots \cup P_{i+1}, & \text { if } i<n ; \\
\varnothing, & \text { if } i=n
\end{array} .\right.
$$

(b) If $S \in J(P)$, then $\left(\mathbf{w}_{0}(S), \mathbf{w}_{1}(S), \ldots, \mathbf{w}_{n}(S)\right)=(0,0, \ldots, 0)$ unless $S$ is level.

Now, we can define a (toylike, but rather useful) notion of homogeneous equivalence, in somewhat questionable analogy with Definition 45.

Definition 95. Two order ideals $S$ and $T$ of $P$ are said to be homogeneously equivalent if and only if either both $S$ and $T$ are level or we have $S=T$. Clearly, being homogeneously equivalent is an equivalence relation. Let $\overline{J(P)}$ denote the set of equivalence classes of elements of $J(P)$ modulo this relation. Let $\pi$ denote the canonical projection $J(P) \rightarrow$ $\overline{J(P)}$. (We distinguish this map $\pi$ from the map $\pi$ defined in Definition 45 by the fact that they act on different objects.)

The following analogue of Corollary 50 is almost trivial:

Corollary 96. If $S$ and $T$ are two homogeneously equivalent order ideals of $P$, then $\mathbf{r}(S)$ is homogeneously equivalent to $\mathbf{r}(T)$.

(An analogue of Corollary 49 exists as well.) We also have the following analogue of Proposition 54:

Proposition 97. Let $S$ and $T$ be two order ideals of $P$ such that $\pi(S)=\pi(T)$ and $\left(\mathbf{w}_{0}(S), \mathbf{w}_{1}(S), \ldots, \mathbf{w}_{n}(S)\right)=\left(\mathbf{w}_{0}(T), \mathbf{w}_{1}(T), \ldots, \mathbf{w}_{n}(T)\right)$. Then, $S=T$.

We can furthermore state analogues of Definitions 52 and 53: 
Definition 98. Fix $i \in\{1,2, \ldots, n\}$. The map $\mathbf{r}_{i}: J(P) \rightarrow J(P)$ descends (through the projection $\pi: J(P) \rightarrow \overline{J(P)}$ ) to a map $\overline{J(P)} \rightarrow \overline{J(P)}$. We denote this map $\overline{J(P)} \rightarrow \overline{J(P)}$ by $\overline{\mathbf{r}_{i}}$. Thus, the diagram

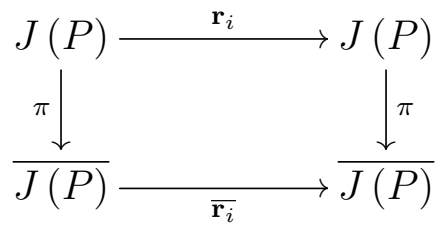

is commutative.

Definition 99. Define the map $\overline{\mathbf{r}}: \overline{J(P)} \rightarrow \overline{J(P)}$ by

$$
\overline{\mathbf{r}}=\overline{\mathbf{r}_{1}} \circ \overline{\mathbf{r}_{2}} \circ \ldots \circ \overline{\mathbf{r}_{n}} .
$$

Then, the following diagram commutes:

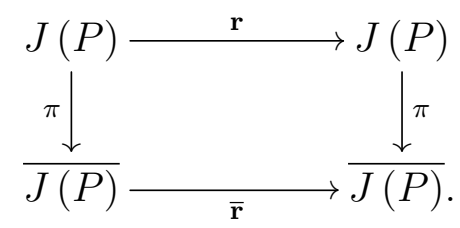

It might seem that the map $\overline{\mathbf{r}}$ is not worth considering, since its cycle structure differs from the cycle structure of $\mathbf{r}$ only in the collapsing of an $(n+1)$-cycle (the one formed by all level order ideals) to a point. However, triviality in combinatorics does not preclude usefulness, and we will employ the "projective" version $\overline{\mathbf{r}}$ of classical rowmotion as a stirrup in determining the order of classical rowmotion $\mathbf{r}$ on skeletal posets.

We have the following simple relation between the orders of $\mathbf{r}$ and $\overline{\mathbf{r}}$ :

Proposition 100. Let $P$ be an n-graded poset. Then, ord $\mathbf{r}=\operatorname{lcm}(n+1, \operatorname{ord} \overline{\mathbf{r}})$.

Proof of Proposition 100. We know that $\mathbf{r}$ is an invertible map $J(P) \rightarrow J(P)$, thus a permutation of the finite set $J(P)$. Hence, ord $\mathbf{r}$ is the lcm of the lengths of the cycles of this permutation $\mathbf{r}$. Similarly, ord $\overline{\mathbf{r}}$ is the $\mathrm{lcm}$ of the lengths of the cycles of the permutation $\overline{\mathbf{r}}$ of the finite set $\overline{J(P)}$. Therefore, in order to prove Proposition 100, it is enough to show that there exists a 1-to-1 correspondence between the cycles of $\mathbf{r}$ and the cycles of $\overline{\mathbf{r}}$ which preserves the length of a cycle except for one particular length- $(n+1)$ cycle of $\mathbf{r}$ which it maps to a 1-cycle of $\overline{\mathbf{r}}$.

Such a correspondence is easy to find: The set $\overline{J(P)}$ is the quotient of $J(P)$ modulo homogeneous equivalence. But homogeneous equivalence merely identifies the $n+1$ level order ideals (which form a cycle under $\mathbf{r}$ ), while all other elements of $J(P)$ are pairwise non-equivalent. Thus, the cycles of $\overline{\mathbf{r}}$ are in 1-to-1 correspondence with the cycles of $\mathbf{r}$, and corresponding cycles have equal lengths except for the length- $(n+1)$ cycle formed by the $n+1$ level order ideals in $J(P)$ and its corresponding length-1 cycle in $\overline{J(P)}$. This is the correspondence we were looking for. 
Our goal is to make a statement about the order of classical rowmotion on skeletal posets. Of course, the finiteness of these orders is obvious in this case, because $J(P)$ is a finite set. However, we can make stronger claims:

Proposition 101. For any skeletal poset $P$, we have ord $\left(R_{P}\right)=\operatorname{ord}\left(\mathbf{r}_{P}\right)$ and ord $\left(\bar{R}_{P}\right)=$ ord $\left(\overline{\mathbf{r}}_{P}\right)$.

Proposition 101 yields (in particular) that the order of classical rowmotion coincides with the order of birational rowmotion (whatever the base field) for skeletal posets. This was conjectured by James Propp (private communication) for the case of $P$ a tree. We are going to prove Proposition 101 by exhibiting further analogies between classical and birational rowmotion. First of all, the following proposition is just as trivial as its birational counterpart (Proposition 72):

Proposition 102. Fix $n \in \mathbb{N}$, and let $P$ and $Q$ be two n-graded posets. Then, ord $\left(\mathbf{r}_{P Q}\right)=$ $\operatorname{lcm}\left(\operatorname{ord}\left(\mathbf{r}_{P}\right)\right.$, ord $\left.\left(\mathbf{r}_{Q}\right)\right)$.

We can show a simple counterpart of this proposition for ord $\left(\overline{\mathbf{r}}_{P Q}\right)$ (but still with ord $\left(\mathbf{r}_{P}\right)$ and ord $\left(\mathbf{r}_{Q}\right)$ on the right hand side!):

Proposition 103. Fix $n \in \mathbb{N}$, and let $P$ and $Q$ be two n-graded posets. Then, ord $\left(\overline{\mathbf{r}}_{P Q}\right)=$ $\operatorname{lcm}\left(\operatorname{ord}\left(\mathbf{r}_{P}\right)\right.$, ord $\left.\left(\mathbf{r}_{Q}\right)\right)$.

Again, the divisibility of the right hand side by the left one is the most useful statement, and it follows trivially from the results before. The opposite divisibility can be obtained by studying the orbit of the order ideal $P$ of $P Q$ (which is not level and thus does not collapse under $\pi$ ). See [GrRoArX, §10] for details.

More interesting is the analogue of Proposition 74, whose proof is also analogous, though easier (full details in [GrRoArX]).

Proposition 104. Let $n \in \mathbb{N}$. Let $P$ be an $n$-graded poset.

(a) We have ord $\left(\overline{\mathbf{r}}_{B_{1} P}\right)=$ ord $\left(\overline{\mathbf{r}}_{P}\right)$.

(b) For every integer $k>1$, we have ord $\left(\overline{\mathbf{r}}_{B_{k} P}\right)=\operatorname{lcm}\left(2\right.$, ord $\left.\left(\overline{\mathbf{r}}_{P}\right)\right)$.

We can also formulate an analogue of Proposition 75:

Proposition 105. Let $P$ be an n-graded poset.

(a) We have ord $\left(\overline{\mathbf{r}}_{B_{1}^{\prime} P}\right)=$ ord $\left(\overline{\mathbf{r}}_{P}\right)$.

(b) For every integer $k>1$, we have ord $\left(\overline{\mathbf{r}}_{B_{k}^{\prime} P}\right)=\operatorname{lcm}\left(2\right.$, ord $\left.\left(\overline{\mathbf{r}}_{P}\right)\right)$.

The proof of this is fairly similar to that of Proposition 104.

We can now prove Proposition 101:

Proof of Proposition 101. In our proof of Proposition 71, we gave an algorithm for inductively computing ord $\left(R_{T}\right)$ and ord $\left(\bar{R}_{T}\right)$ for a skeletal poset $T$. Using Propositions 102105 instead of Propositions 72-75, we obtain the same algorithm for inductively computing ord $\left(\mathbf{r}_{T}\right)$ and ord $\left(\overline{\mathbf{r}}_{T}\right)$ for a skeletal poset $T$. Therefore, ord $\left(R_{P}\right)=\operatorname{ord}\left(\mathbf{r}_{P}\right)$ and $\operatorname{ord}\left(\bar{R}_{P}\right)=\operatorname{ord}\left(\overline{\mathbf{r}}_{P}\right)$. 
Proposition 101 does not generalize to arbitrary graded posets. Counterexamples to such a generalization can be found in [GrRo14, §12].

Finally, in analogy to Corollary 76, we can now show:

Corollary 106. Let $P$ be an n-graded poset that is also a rooted forest (made into a poset by having every node smaller than its children).

(a) Then, ord $\left(\mathbf{r}_{P}\right) \mid \operatorname{lcm}(1,2, \ldots, n+1)$.

(b) Moreover, if $P$ is a tree, then ord $\left(\overline{\mathbf{r}}_{P}\right) \mid \operatorname{lcm}(1,2, \ldots, n)$.

Corollary 106 is also valid if we replace "every node smaller than its children" by "every node larger than its children", and the proof is exactly analogous.

Let us notice that the algorithm described in the proof of Proposition 101 can be turned into an explicit formula (not just an upper bound as in Corollary 106), whose inductive proof we leave to the reader:

Proposition 107. Let $P$ be an n-graded poset that is also a rooted forest (made into a poset by having every node smaller than its children). Notice that $\left|\widehat{P}_{i}\right| \leqslant\left|\widehat{P}_{i+1}\right|$ for every $i \in\{0,1, \ldots, n-1\}$. Then,

$$
\operatorname{ord}\left(\overline{\mathbf{r}}_{P}\right)=\operatorname{lcm}\left\{n-i|i \in\{0,1, \ldots, n-1\} ;| \widehat{P}_{i}|<| \widehat{P}_{i+1} \mid\right\} \text {. }
$$

Of course, ord $\left(\mathbf{r}_{P}\right)$ can now be computed by $\operatorname{ord}\left(\mathbf{r}_{P}\right)=\operatorname{lcm}\left(n+1, \operatorname{ord}\left(\overline{\mathbf{r}}_{P}\right)\right)$.

The same property therefore holds for birational rowmotion $R_{P}$ and its homogeneous version $\bar{R}_{P}$.

\section{Acknowledgments}

The notion of birational rowmotion is due to James Propp and Arkady Berenstein. This paper owes James Propp also for a constant flow of inspiration and useful suggestions.

Hugh Thomas corrected slips in our writing including an abuse of Zariski topology and some accidental alternative history. Nathan Williams noticed further typos.

The first author came to know birational rowmotion in Alexander Postnikov's combinatorics pre-seminar at MIT. Postnikov also suggested veins of further study.

Jessica Striker helped the first author understand some of the past work on this subject, in particular the labyrinthine connections between related operators (rowmotion, Panyushev complementation, Striker-Williams promotion, Schützenberger promotion, etc.). The present paper explores merely one corner of this labyrinth (the rowmotion corner).

We thank the two referees of our FPSAC abstract [GrRo13] for further helpful comments. We also owe a number of improvements in this paper to the suggestions of an anonymous EJC referee.

Both authors were partially supported by NSF grant \#1001905, and have utilized the open-source CAS Sage $\left(\left[\mathrm{S}^{+} 09\right]\right.$, [Sage08]) to perform laborious computations. We thank Travis Scrimshaw, Frédéric Chapoton, Viviane Pons and Nathann Cohen for reviewing Sage patches relevant to this project. 


\section{References}

[AKSch12] Arvind Ayyer, Steven Klee, Anne Schilling, Combinatorial Markov chains on linear extensions, J. Alg. Combin., September 2013, DOI 10.1007/s10801-013-0470-9. Also at arXiv:1205.7074v3.

[BrSchr74] Andries E. Brouwer and A. Schrijver, On the period of an operator, defined on antichains, Math. Centr. report ZW24, Amsterdam (Jun. 1974).

http://www.win.tue.nl/ aeb/preprints/zw24.pdf

[CaFl95] Peter J. Cameron, Dmitry G. Fon-der-Flaass, Orbits of Antichains Revisited, Europ. J. Combin., vol. 16, Issue 6, November 1995, pp. 545-554. http://www.sciencedirect.com/science/article/pii/0195669895900365.

[EiPr13] David Einstein, James Propp, Combinatorial, piecewise-linear, and birational homomesy for products of two chains, arXiv:1310.5294v1 (preliminary version), October 20, 2013.

[EiPr14] David Einstein, James Propp, Piecewise-linear and birational toggling, arXiv:1404.3455v1, DMTCS proc. FPSAC 2014.

[Etienn84] Gwihen Etienne, Linear extensions of finite posets and a conjecture of $G$. Kreweras on permutations, Discrete Mathematics, Volume 52, Issue 1, 1984, pp. $107-111$.

http://www.sciencedirect.com/science/article/pii/0012365X84901080

[Flaa93] Dmitry G. Fon-der-Flaass, Orbits of Antichains in Ranked Posets, Europ. J. of Combin., vol. 14, Issue 1, January 1993, pp. 17-22. http://www.sciencedirect. com/science/article/pii/S0195669883710036.

[GrRoArX] Darij Grinberg, Tom Roby, Iterative properties of birational rowmotion, arXiv:1402.6178v3, 2014. (Full version with detailed proofs.)

[GrRo13] Darij Grinberg, Tom Roby, The order of birational rowmotion, DMTCS proc. FPSAC 2014. (Extended abstract of this paper.)

http://www.dmtcs.org/dmtcs-ojs/index.php/proceedings/article/ viewArticle/dmAT0165.

[GrRo14] Darij Grinberg, Tom Roby, Iterative properties of birational rowmotion II: rectangles and triangles, Electronic J. of Combin., 22(3), 2015, \#P3,40. http: //www. combinatorics.org/ojs/index.php/eljc/article/view/v22i3p40.

[Haiman92] Mark D. Haiman, Dual equivalence with applications, including a conjecture of Proctor, Discrete Mathematics, Volume 99, Issues 1-3, 2 April 1992, pp. 79-113. http://www.sciencedirect.com/science/article/pii/0012365X9290368P

[OSZ13] Neil O'Connell, Timo Seppäläinen, Nikos Zygouras, Geometric RSK correspondence, Whittaker functions and symmetrized random polymers, Invent. Math., October 2013, DOI 10.1007/s00222-013-0485-9. http://dx.doi.org/10. 1007/s00222-013-0485-9. An older preprint version at arXiv:1210.5126v2. 
[PrRo13] James Propp and Tom Roby, Homomesy in products of two chains, Electronic J. of Combin., 22(3), 2015, \#P3,4. http://www.combinatorics.org/ojs/index. php/eljc/article/view/v22i3p4. Also, arXiv:arXiv:1310.5201v5.

[Rusk92] Frank Ruskey, Generating Linear Extensions of Posets by Transpositions, J. of Combin. Thy, vol. 54, Issue 1, January 1992, pp. 77-101. http://www . sciencedirect.com/science/article/pii/0095895692900678

[S+09] W. A. Stein et al., Sage Mathematics Software (Version 6.2.beta2), The Sage Development Team, 2014, http://www. sagemath.org.

[Sage08] The Sage-Combinat community, Sage-Combinat: enhancing Sage as a toolbox for computer exploration in algebraic combinatorics, 2008. http://combinat.sagemath.org.

[Stan11] Richard P. Stanley, Enumerative Combinatorics, volume 1, 2nd edition, no. 49 in Cambridge Studies in Advanced Mathematics, Cambridge University Press, 2011. Also available at http://math.mit.edu/ rstan/ec/ec1/.

[Stan86] Richard Stanley, Two Poset Polytopes, Disc. \& Comp. Geom., 1986, Volume 1, Issue 1, pp. 9-23.

[StWi11] Jessica Striker, Nathan Williams, Promotion and Rowmotion, Europ. J. of Combin. 33 (2012), pp. 1919-1942, DOI 10.1016/j.ejc.2012.05.003.

http://www.sciencedirect.com/science/article/pii/S0195669812000972.

Also available as arXiv:1108.1172v3. 
Corrigendum - Added Apr 2, 2023

Bruce Sagan has kindly informed us of an error in Proposition 107: The $n-i$ on the right hand side has to be replaced by $n+1-i$. A proof of the corrected proposition can be found in the updated arXiv postprint version (https://arxiv.org/abs/1402.6178v7). 